\title{
DIMENSIONS OF SOME LOCALLY ANALYTIC REPRESENTATIONS
}

\author{
TOBIAS SCHMIDT AND MATTHIAS STRAUCH
}

\begin{abstract}
Let $G$ be the group of points of a split reductive group over a finite extension of $\mathbb{Q}_{p}$. In this paper, we compute the dimensions of certain classes of locally analytic $G$-representations. This includes principal series representations and certain representations coming from homogeneous line bundles on $p$-adic symmetric spaces. As an application, we compute the dimensions of the unitary $\mathrm{GL}_{2}\left(\mathbb{Q}_{p}\right)$-representations appearing in Colmez' $p$-adic local Langlands correspondence.
\end{abstract}

\section{Contents}

1. Introduction

2. Grade and dimension

3. Arens-Michael envelopes and faithful flatness

4. From $D(\mathfrak{g}, P)$-modules to $D(G)$-modules

5. Highest weight modules and dimension

6. Application to equivariant line bundles on Drinfeld's upper half space

7. Dimensions for $\mathrm{GL}_{2}\left(\mathbb{Q}_{p}\right)$ and $p$-adic Galois representations

Acknowledgments

References

\section{INTRODUCTION}

Let $L$ be a finite extension of $\mathbb{Q}_{p}$ and let $G=\mathbf{G}(L)$ be the group of $L$-valued points of a split connected reductive algebraic group $\mathbf{G}$ over $L$. Let $P \subseteq G$ be a parabolic subgroup.

Admissible Banach space representations or locally analytic representations of $G$ admit a well-behaved notion of (canonical) dimension. The rational representations coming from the algebraic group $\mathbf{G}$ or the traditional smooth representations from Langlands theory are known to have dimension zero. Moreover, any representation which is not zero-dimensional has dimension greater or equal to half the dimension of the minimal nilpotent orbit of $\mathbf{G}[3$. Besides these general results, the dimensions of even very explicit representations like principal series representations have

Received by the editors December 18, 2014 and, in revised form, November 17, 2015 and December 11, 2015.

2010 Mathematics Subject Classification. Primary 11E95, 22E50; Secondary 11S80, 16S30.

The first author would like to acknowledge support of the Heisenberg programme of Deutsche Forschungsgemeinschaft.

The second author would like to acknowledge the support of the National Science Foundation (award DMS-1202303). 
not been computed so far. In this paper, we make an attempt to close this gap and determine the dimensions of certain families of representations. This includes principal series representations as well as representations coming from $p$-adic symmetric spaces. The technical key result is that the functor $\mathcal{F}_{P}^{G}$ introduced by Orlik and the second author in [28] from Lie algebra representations of $\mathfrak{g}=\operatorname{Lie}(G)$ endowed with a compatible action of $P$ to locally analytic $G$-representations preserves the dimension.

As an application, we compute the dimensions in Colmez' $p$-adic local Langlands correspondence 12 for $\mathrm{GL}_{2}\left(\mathbb{Q}_{p}\right)$. Let $\Pi(V)$ denote the unitary representation of $\mathrm{GL}_{2}\left(\mathbb{Q}_{p}\right)$ corresponding to an absolutely irreducible 2-dimensional $p$-adic Galois representation $V$ of $\operatorname{Gal}\left(\overline{\mathbb{Q}}_{p} / \mathbb{Q}_{p}\right)$. We show that the resulting map $V \mapsto \operatorname{dim} \Pi(V)$ is constant with value 1 .

In the following we give more details on the individual sections of this paper. In section 2 we review basic notions of dimension theory and establish two auxiliary lemmas. In section 3 we develop a framework which allows us to prove faithful flatness of Arens-Michael envelopes in many situations. In section 4 we combine this result, in the case of the universal enveloping algebra $U(\mathfrak{g})$ of $\mathfrak{g}=\operatorname{Lie}(G)$, with a study of the functor $\mathcal{F}_{P}^{G}$ and prove that the latter preserves dimensions. On the level of Lie algebra representations, canonical dimension coincides with the more traditional Gelfand-Kirillov dimension and this enables us to give explicit dimension formulas for the representations $\mathcal{F}_{P}^{G}(M)$ whenever the Gelfand-Kirillov dimension for $M$ (viewed as an $U(\mathfrak{g})$-module) is known. We illustrate this in section 5 in the case of the classical parabolic Bernstein-Gelfand-Gelfand category for $\mathfrak{p} \subseteq \mathfrak{g}$ where $\mathfrak{p}=\operatorname{Lie}(P)$. For example, the dimension of the locally analytic parabolic induction $\operatorname{Ind}_{P}^{G}(V)$ where $V$ is a locally analytic $P$-representation on a finite-dimensional vector space equals the vector space dimension of $\mathfrak{g} / \mathfrak{p}$. We also remark that the dimensions of irreducible objects in the BGG-category can be computed out of the Kazhdan-Lusztig conjecture through Joseph's Goldie rank polynomials. The main result of [28] shows that the functor $\mathcal{F}_{P}^{G}$ preserves irreducibility in many cases which yields the dimensions of all the irreducible $G$-representations which can be constructed through a functor of type $\mathcal{F}_{P}^{G}$. In section 6 we let $G=\mathrm{GL}_{d+1}(L)$ and compute the dimension of locally analytic representations coming from homogeneous line bundles on Drinfeld's upper half space [27]. In section 7 we give the aforementioned application to $\mathrm{GL}_{2}\left(\mathbb{Q}_{p}\right)$.

Notation and conventions: We denote by $p$ a prime number and consider fields $L \subset K$ which are both finite extensions of $\mathbb{Q}_{p}$. Let $o_{L}$ and $o_{K}$ be the rings of integers of $L$, resp. $K$, and let $|\cdot|_{K}$ be the absolute value on $K$ such that $|p|_{K}=p^{-1}$. The field $L$ is our "base field", whereas we consider $K$ as our "coefficient field". For a locally convex $K$-vector space $V$ we denote by $V_{b}^{\prime}$ its strong dual, i.e., the $K$-vector space of continuous linear forms equipped with the strong topology of bounded convergence. Sometimes, in particular, when $V$ is finite-dimensional, we simplify notation and write $V^{\prime}$ instead of $V_{b}^{\prime}$. All finite-dimensional $K$-vector spaces are equipped with the unique Hausdorff locally convex topology.

We let $\mathbf{G}$ be a split reductive group scheme over $o_{L}$ and $\mathbf{T} \subset \mathbf{B} \subset \mathbf{G}$ a maximal split torus and a Borel subgroup scheme, respectively. We denote the base change to $L$ of these group schemes by the same letters. We let $\mathbf{B} \subseteq \mathbf{P}$ be a parabolic subgroup and let $\mathbf{L}_{\mathbf{P}}$ be the unique Levi subgroup which contains $\mathbf{T}$. By $G_{0}=\mathbf{G}\left(o_{L}\right)$, $B_{0}=\mathbf{B}\left(o_{L}\right)$, etc., and $G=\mathbf{G}(L), B=\mathbf{B}(L)$, etc., we denote the corresponding 
groups of $o_{L}$-valued points and $L$-valued points, respectively. Finally, Gothic letters $\mathfrak{g}, \mathfrak{p}$, etc., usually denote the Lie algebras of $G, P$, etc.: $\mathfrak{g}=\operatorname{Lie}(G), \mathfrak{t}=\operatorname{Lie}(T)$, $\mathfrak{b}=\operatorname{Lie}(B), \mathfrak{p}=\operatorname{Lie}(P), \mathfrak{l}_{\mathfrak{p}}=\operatorname{Lie}\left(L_{P}\right)$, etc. A base change to $K$ is usually denoted by the subscript ${ }_{K}$, for instance, $\mathfrak{g}_{K}=\mathfrak{g} \otimes_{L} K$.

\section{GRADE AND DIMENSION}

In this section we introduce some basic notions in dimension theory and establish two simple lemmas. The term module always means left module. Noetherian rings are two-sided noetherian and other ring-theoretic properties are used similarly.

We recall the notion of an Auslander regular ring 23. Let $R$ be an arbitrary associative unital ring. For any $R$-module $N$ the grade $j_{R}(N)$ is defined to be either the smallest integer $k$ such that $\operatorname{Ext}_{R}^{k}(N, R) \neq 0$ or $\infty$. Now suppose that $R$ is (left and right) noetherian. If $N \neq 0$ is finitely generated, then its grade $j_{R}(N)$ is bounded above by the projective dimension of $N$. A noetherian ring $R$ is called Auslander regular if its global dimension is finite and if every finitely generated $R$-module $N$ satisfies Auslander's condition: for any $k \geqslant 0$ and any $R$-submodule $L \subseteq \operatorname{Ext}_{R}^{k}(N, R)$ one has $j_{R}(L) \geqslant k$.

Let $R$ be an Auslander regular ring of finite global dimension $\operatorname{gld}(R)$ and $M$ an $R$-module. The number

$$
\operatorname{dim}_{R} M:=\operatorname{gld}(R)-j_{R}(M)
$$

is called the canonical dimension of $M$. One has

$$
\operatorname{dim}_{R} M=\max \left\{\operatorname{dim}_{R} M^{\prime}, \operatorname{dim}_{R} M^{\prime \prime}\right\}
$$

for an exact sequence $0 \rightarrow M^{\prime} \rightarrow M \rightarrow M^{\prime \prime} \rightarrow 0$. Moreover, $\operatorname{dim}_{R} 0=-\infty$.

Let $\tau$ be an automorphism of $R$ and let $M$ be a left $R$-module. We denote by ${ }^{\tau} M$ the abelian group $M$ with the left $R$-action $r . m:=\tau(r) m$ and call ${ }^{\tau} M$ the twist of $M$ with $\tau$. In the case of a right module $M$ we denote the analogous construction by $M^{\tau}$.

Lemma 2.1. Twisting with $\tau$ has the following properties:

(i) the functor $M \rightarrow^{\tau} M$ is an auto-equivalence on the category of all $R$ modules,

(ii) $M$ is finitely generated if and only if ${ }^{\tau} M$ is finitely generated,

(iii) there are canonical isomorphisms $\operatorname{Ext}_{R}^{k}(\tau, M, R) \simeq \operatorname{Ext}_{R}^{k}(M, R)^{\tau}$ for all $k$,

(iv) one has $j_{R}(M)=j_{R}\left({ }^{\tau} M\right)$.

Proof. Twisting with $\tau^{-1}$ yields a quasi-inverse, so (i) is clear. (ii) is trivial so let us turn to (iii). In the case of $k=0$ the isomorphism is given explicitly by sending a linear form $f$ on ${ }^{\tau} M$ to the linear form $\tau \circ f$ on $M$. According to (i) a projective resolution $P$ • for $M$ yields a projective resolution ${ }^{\tau} P$ • for ${ }^{\tau} M$. Since the isomorphism for $k=0$ is natural in $M$, we are done. (iv) follows formally from (iii).

Lemma 2.2. Let $R \rightarrow S$ be a faithfully flat ring extension between noetherian rings. Let $M$ be a finitely generated $R$-module. We have

$$
j_{S}\left(S \otimes_{R} M\right)=j_{R}(M) .
$$


Proof. Put $M_{S}:=S \otimes_{R} M$. We have $\operatorname{Ext}_{R}^{k}(M, R) \otimes_{R} S \simeq \operatorname{Ext}_{S}^{k}\left(M_{S}, S\right)$ for all $k$. Indeed, since $R \rightarrow S$ is flat, choosing a free resolution of $M$ by finitely generated free modules reduces us to the case $k=0$ and $M=R$ where the statement is obvious. By faithful flatness of $R \rightarrow S$, we have $\operatorname{Ext}_{R}^{j_{R}(M)}(M, R) \otimes_{R} S \neq 0$ which implies the claim.

\section{Arens-Michael envelopes and faithful Flatness}

Let $R$ be a complete discrete valuation ring with field of fractions $K$ and uniformizer $\pi$.

Let $A$ be an $R$-algebra, flat as an $R$-module, which is $\pi$-adically separated, that is, $\bigcap_{m \geqslant 0} \pi^{m} A=0$. Let $A$ be equipped with an increasing and exhaustive filtration

$$
F_{0} A \subseteq F_{1} A \subseteq F_{2} A \subseteq \ldots
$$

by $R$-submodules such that $1 \in F_{0} A$ and $F_{i} A \cdot F_{j} A \subseteq F_{i+j} A$ for all $i, j$. In particular, $F_{0} A$ is an $R$-subalgebra of $A$. We make the following two assumptions on this filtration:

(1) The ring $F_{0} A$ is a commutative noetherian integral domain such that $F_{0} A$ / $\pi F_{0} A$ is a regular integral domain;

(2) The associated graded $\operatorname{ring} g r_{\bullet}^{F} A$ is commutative and isomorphic to a polynomial ring over $F_{0} A$ in finitely many, say $r$, variables (where the polynomial ring has its usual positive grading by total degree with the variables placed in degree one).

The regularity assumption in (1) means that all local rings of $F_{0} A / \pi F_{0} A$ at prime ideals are regular or, equivalently, that the ring $F_{0} A / \pi F_{0} A$ has finite global dimension. Of course, any filtration with $F_{0} A=R$ satisfies (1), but there is no point in restricting to this special case at the moment. We also note that (2) implies by induction on $i+j$ :

(3) We have $F_{i} A \cdot F_{j} A=F_{j} A \cdot F_{i} A$ as $R$-submodules of $A$ for all $i, j$.

Indeed, the principal symbol in $g_{i+j}^{F} A$ of some element $x \in F_{i+j} A \backslash F_{i+j-1} A$ is a finite sum of nonzero products $y_{i} y_{j}$ with $y_{i} \in g r_{i}^{F} A, y_{j} \in g r_{j}^{F} A$. If $x_{k} \in F_{k} A$ is a lift of $y_{k}$, then, by induction, we may assume that the difference $x-\sum_{i, j} x_{i} x_{j} \in F_{i+j-1}$ lies in $F_{i} A \cdot F_{j-1} A$. Hence $x \in F_{i} A \cdot F_{j} A$ as required.

Positively filtered algebras $A$ that satisfy these requirements abound. The main examples we have in mind are universal enveloping algebras of Lie algebras as well as the rings of (crystalline) differential operators on certain smooth affine $R$-schemes. We will give more details at the end of this section.

In the following we will assume that these conditions hold. We then have the $K$-algebras

$$
\left(F_{0} A\right)_{K}:=F_{0} A \otimes_{R} K \quad \text { and } \quad A_{K}:=A \otimes_{R} K .
$$

The algebra $\left(F_{0} A\right)_{K}$ has a natural structure of normed algebra by declaring the lattice $F_{0} A$ to be the unit ball. We give $A_{K}$ the finest locally convex topology making the inclusion map $\left(F_{0} A\right)_{K} \hookrightarrow A_{K}$ continuous (where the source has its norm topology). Our aim in this subsection is to analyze the algebraic and homological properties of the Arens-Michael envelope $\hat{A}_{K}$ of the locally convex algebra $A_{K}$. 
Recall $[15]^{1}$ that

$$
\begin{aligned}
\hat{A}_{K}:= & \text { (Hausdorff) completion of } A_{K} \text { w.r.t. all continuous } \\
& \text { submultiplicative seminorms. }
\end{aligned}
$$

Among our main results will be that $\hat{A}_{K}$ is a Fréchet-Stein algebra in the sense of [41] and that the canonical completion homomorphism $A_{K} \rightarrow \hat{A}_{K}$ is a faithfully flat ring extension. As we will see, these results make the homological algebra of $\hat{A}_{K}$ quite transparent.

As a first step we will obtain a more accessible description of $\hat{A}_{K}$. To this end, we consider the Rees ring

$$
R_{\bullet}^{F}(A):=\bigoplus_{i \geqslant 0}\left(F_{i} A\right) X^{i}
$$

of the filtered ring $A$, viewed as a subring of the polynomial ring $A[X]$. The ring $R_{\bullet}^{F}(A)$ is noetherian according to [23, II.2.2.1]. For each number $n \geqslant 0$ we let $A_{n}$ be the image of $R_{\bullet}^{F}(A)$ under the evaluation homomorphism $A[X] \rightarrow A$ given by $X \mapsto \pi^{n}$. Obviously, $A_{n+1} \subseteq A_{n}$ and $A_{0}=A$. Let $\hat{A}_{n}$ be the $\pi$-adic completion of $A_{n}$ and put $\hat{A}_{n, K}:=\hat{A}_{n} \otimes_{R} K$. All rings $A_{n}, \hat{A}_{n}$ and $\hat{A}_{n, K}$ are noetherian. Indeed, $A_{n}$ is a homomorphic image of the noetherian Rees ring and $\hat{A}_{n}$ is its adic completion at a central element [5, 3.2.3]. Finally, $\hat{A}_{n, K}$ is a localization of $\hat{A}_{n}$ and hence noetherian, too.

In the following we will need some basic results on the interplay between the positive filtration $F_{\bullet} A$ on $A$, the $\pi$-adic filtration on $A$ and the rings $A_{n}$. Such results are established by K. Ardakov and S. Wadsley in [3] and to be completely clear, we therefore relate our situation to the terminology used in loc. cit. The positively filtered ring $A$ is an almost commutative $R$-algebra in the sense of the definition [3, 3.4]. Moreover, it is deformable and $A_{n}$ is its $n$-th deformation [3, 3.5]. According to [3, Prop. 3.8] the algebra $\hat{A}_{n, K}$ is therefore an almost commutative affinoid $K$-algebra in the sense of the definition [3, 3.8]. In particular, $\hat{A}_{n, K}$ is a complete doubly filtered $K$-algebra with slice $\hat{A}_{n} / \pi \hat{A}_{n}[3,3.1]$. Of course, we have $A_{n} / \pi A_{n}=\hat{A}_{n} / \pi \hat{A}_{n}$.

Each ring $A_{n}$ has its induced filtration $F_{m} A_{n}:=A_{n} \cap F_{m} A$. Since $g r_{\bullet}^{F} A$ is flat over $R$ one has

$$
F_{m} A_{n}=\sum_{i=0, \ldots, m} \pi^{i n} F_{i} A
$$

In particular, $F_{0} A_{n}=F_{0} A$. The graded ring $g r_{.}^{F} A_{n}$ is in fact isomorphic to the graded ring $g r_{\bullet}^{F} A$ via the map given on the $i$-th homogeneous component as

$$
F_{i} A / F_{i-1} A \longrightarrow F_{i} A_{n} / F_{i-1} A_{n}, \quad x+F_{i-1} A \mapsto \pi^{i n} x+F_{i-1} A_{n},
$$

[3. Lem. 3.5]. In particular, $g r_{\bullet}^{F} A_{n}$ is isomorphic, as a graded ring, to a polynomial ring in $r$ variables over $F_{0} A_{n}$.

The slice $A_{n} / \pi A_{n}$ has the quotient filtration coming from $F_{\bullet} A_{n}$ and we let $g r_{\bullet}^{F}\left(A_{n} / \pi A_{n}\right)$ be the associated graded ring. According to [3, Lem. 3.7] the map $A_{n} \rightarrow A_{n} / \pi A_{n}$ induces an isomorphism of graded rings

\footnotetext{
${ }^{1}$ The classical notion of an Arens-Michael envelope [15] is given for complex algebras, but the definition extends readily to any valued field.
} 


$$
g r_{\bullet}^{F} A_{n} / \pi g r_{\bullet}^{F} A_{n} \stackrel{\simeq}{\longrightarrow} g r_{\bullet}^{F}\left(A_{n} / \pi A_{n}\right)
$$

Following [3, 3.1] we finally abbreviate

$$
\operatorname{Gr}\left(\hat{A}_{n, K}\right):=g r_{\bullet}^{F}\left(A_{n} / \pi A_{n}\right) .
$$

This is a polynomial ring over $F_{0} A_{n} / \pi F_{0} A_{n}$ in $r$ variables and is therefore a noetherian regular integral domain according to (1).

Proposition 3.4. The homomorphism $\hat{A}_{n+1, K} \rightarrow \hat{A}_{n, K}$ is flat for all $n$.

Proof. We follow an overall strategy of Berthelot [5, 3.5.3] which is made explicit by Emerton in [14, 5.3.10]. In particular, we want to apply the following statement (loc. cit.). Let $C$ be a (not necessarily commutative) $p$-torsion free and $p$-adically separated left noetherian $\mathbb{Z}_{p}$-algebra. If $B$ is a $\mathbb{Z}_{p}$-subalgebra of $\mathbb{Q}_{p} \otimes_{\mathbb{Z}_{p}} C$ that contains $C$ and that is equipped with an exhaustive increasing filtration by $\mathbb{Z}_{p^{-}}$ submodules $F_{0} \subset F_{1} \subset \cdots$ satisfying the following assumptions:

(i) for each pair $i, j$ we have $F_{i} \cdot F_{j} \subset F_{i+j}$;

(ii) $F_{0}=C$;

(iii) the associated graded algebra of $B$ is finitely generated over $A$ by central elements,

then $\mathbb{Q}_{p} \otimes_{\mathbb{Z}_{p}} B$ is flat as a right $\mathbb{Q}_{p} \otimes_{\mathbb{Z}_{p}} C$-module.

We will apply this statement to the rings $C:=A_{n+1}$ and $B:=A_{n}$ where the filtration on $B=A_{n}$ will be a certain 'augmented' filtration which we now define. Let

$$
F_{m}^{\prime} A_{n}:=A_{n+1} \cdot F_{m} A_{n}
$$

for all $m$. We claim that this filtration satisfies

$$
F_{k}^{\prime} A_{n} \cdot F_{\ell}^{\prime} A_{n} \subseteq F_{k+\ell}^{\prime} A_{n}
$$

for all $k, \ell$ so that we have an associated graded $\operatorname{ring} g r r^{F^{\prime}} A_{n}$. To prove the claim, it suffices to verify

$$
A_{n+1} \cdot F_{m} A_{n}=F_{m} A_{n} \cdot A_{n+1} .
$$

Because of $A_{n+1}=\sum_{j \geqslant 0} \pi^{(n+1) j} F_{j} A$ together with (3.1) this reduces to

$$
\pi^{(n+1) j} F_{j} A \cdot \pi^{i n} F_{i} A=\pi^{i n} F_{i} A \cdot \pi^{(n+1) j} F_{j} A
$$

for each $i, j$. However, this is a direct consequence of (3). Secondly, we observe that $F_{0} A_{n}=F_{0} A$ which implies $g r_{0}^{F^{\prime}} A_{n}=F_{0}^{\prime} A_{n}=A_{n+1}$. Finally, we claim that the ring $g r_{\bullet}^{F^{\prime}} A_{n}$ is finitely generated over $g r_{0}^{F^{\prime}} A_{n}$ by central elements. To start with, the composite

$$
F_{m+1} A_{n} \subseteq F_{m+1}^{\prime} A_{n} \rightarrow F_{m+1}^{\prime} A_{n} / F_{m}^{\prime} A_{n}
$$

is surjective and factors through $F_{m+1} A_{n} / F_{m} A_{n}$ for all $m \geqslant 0$." We obtain a graded ring homomorphism

$$
f: g r_{\bullet}^{F} A_{n} \rightarrow g r_{\bullet}^{F^{\prime}} A_{n}
$$

whose image equals $F_{0} A_{n} \oplus\left(\oplus_{m>0} g r_{m}^{F^{\prime}} A_{n}\right)$ with $F_{0} A_{n} \subset g r_{0}^{F^{\prime}} A_{n}$. According to the isomorphism (3.2) and our hypothesis (2) on $g r_{\bullet}^{F} A$, the source of $f$ is a polynomial ring over $F_{0} A_{n}$ in finitely many variables, say $y_{1}, \ldots, y_{r} \in g r_{1}^{F} A_{n}$. It therefore suffices to see that the images of these generators in $g r_{\bullet}^{F^{\prime}} A_{n}$ are central, that is, 
they commute with $g r_{0}^{F^{\prime}} A_{n}=F_{0}^{\prime} A_{n}=A_{n+1}$. To this end, we choose elements $x_{1}, \ldots, x_{r}$ in $F_{1} A$ such that $y_{i}=\pi^{n} x_{i}+F_{0} A_{n}$. This is possible according to (3.2). The commutator $\left[g r_{0}^{F^{\prime}} A_{n}, f\left(y_{i}\right)\right]$ vanishes in $g r_{\bullet}^{F^{\prime}} A_{n}$, if we can show the inclusion $\left[A_{n+1}, \pi^{n} x_{i}+F_{0} A_{n}\right] \subseteq A_{n+1}$ inside $A_{n}$. Since $F_{0} A_{n}=F_{0} A_{n+1} \subset A_{n+1}$ and since $\left[\cdot, \pi^{n} x_{i}\right]$ is additive, we are reduced to show

$$
\left[\pi^{(n+1) j} z, \pi^{n} x_{i}\right] \in A_{n+1}
$$

for any $z \in F_{j} A$ and $j \geqslant 0$. Since $g r_{\bullet}^{F} A$ is commutative, the commutator $\left[z, x_{i}\right] \in$ $F_{j+1} A$ lies, in fact, in the subgroup $F_{j} A$. This implies

$$
\left[\pi^{(n+1) j} z, \pi^{n} x_{i}\right]=\pi^{(n+1) j+n}\left[z, x_{i}\right] \in \pi^{n} \cdot \pi^{(n+1) j} F_{j} A \subset \pi^{n} \cdot F_{j} A_{n+1} \subset F_{j} A_{n+1}
$$

which proves the claim. All in all, we have now verified the conditions (i), (ii), (iii) above for the augmented filtration $F^{\prime} A_{n}$ on $A_{n}$ and its subring $F_{0}^{\prime} A_{n}=A_{n+1}$. Hence, we obtain the flatness of $\hat{A}_{n+1, K} \rightarrow \hat{A}_{n, K}$.

The proposition implies that the projective limit

$$
\lim _{n} \hat{A}_{n, K}
$$

with its projective limit topology, is a Fréchet-Stein algebra in the sense of [41].

Proposition 3.5. The canonical map $\varliminf_{n} \hat{A}_{n, K} \stackrel{\simeq}{\rightarrow} \hat{A}_{K}$ is an isomorphism of topological algebras.

Proof. Clearly, any $A_{n}$ gives rise to a continuous submultiplicative seminorm, say $\|.\|_{n}$, on $A_{K}$ and it suffices to see that these are cofinal in the directed set of all such seminorms on $A_{K}$. According to [3, Lem. 3.1], the graded ring of $A_{n}$ relative to its $\pi$-adic filtration is isomorphic to a polynomial ring $\left(A_{n} / \pi A_{n}\right)[t]$ in one variable $t$ over $A_{n} / \pi A_{n}$. Since $\operatorname{Gr}\left(\hat{A}_{n, K}\right)$ is an integral domain, the rings $A_{n} / \pi A_{n}$ and $\left(A_{n} / \pi A_{n}\right)[t]$ are integral domains, too. This implies that $\|\cdot\|_{n}$ is in fact multiplicative. After these preliminaries, we consider an arbitrary continuous and submultiplicative seminorm $\|$.$\| on A_{K}$. Choose a graded isomorphism between $g_{.}^{F} A$ and a polynomial ring over $F_{0} A$ and lift the variables to elements $x_{1}, \ldots, x_{r}$ in $F_{1} A$. By (1) the ring $F_{0} A$ is an integral domain and, hence, so is $g r_{\bullet}^{F} A$. In particular, the principal symbol map for $g_{\bullet}^{F} A$ is multiplicative. It follows that the ordered monomials $\underline{x}^{\underline{k}}:=x_{1}^{k_{1}} \cdots x_{r}^{k_{r}}$ for $\underline{k}:=\left(k_{1}, \ldots, k_{r}\right) \in \mathbb{N}^{r}$ form a basis of the $F_{0} A$-module $A$. Take an element $a \in A_{K}$ and write

$$
a=\sum_{\underline{k}} a_{\underline{k}} \underline{x}^{\underline{k}}
$$

with uniquely determined $a_{\underline{k}} \in\left(F_{0} A\right)_{K}$. Let $\mid$.| be the norm on $\left(F_{0} A\right)_{K}$ and choose $n$ large enough such that ||$x_{i}|| \leqslant|\pi|^{-n}$ for all $i$. By (3.2) the symbols of the elements $\pi^{n} x_{i}$ in $g r_{\bullet}^{F} A_{n}$ are in degree one and constitute a complete set of variables over $F_{0} A_{n}$. Repeating the argument above for $A_{n}$ shows that $\left\|\pi^{n} x_{i}\right\|_{n}=1$ for all $i$ and that

$$
\|a\|_{n}=\max _{\underline{k}}\left|a_{\underline{k}}\right| \cdot \prod_{i}|| x_{i} \|_{n}^{k_{i}}=\max _{\underline{k}}\left|a_{\underline{k}}\right| \cdot \prod_{i}|\pi|^{-n k_{i}} .
$$

Our assertion follows now from

$$
\|a\| \leqslant \max _{\underline{k}}\left|a_{\underline{k}}\right| \cdot\left\|\underline{x}^{\underline{k}}\right\| \leqslant \max _{\underline{k}}\left|a_{\underline{k}}\right| \prod_{i}\left\|x_{i}\right\|^{k_{i}} \leqslant\|a\|_{n} .
$$


Proposition 3.6. Assume that $F_{0} A$ is $\pi$-adically complete. Then canonical homomorphism $A_{K} \rightarrow \hat{A}_{K}$ is faithfully flat.

Before we turn to the proof of the proposition we establish two auxiliary lemmas. We consider the $\pi$-adic filtration on $A_{n}, \hat{A}_{n}$ and $\hat{A}_{n, K}$. Let $g r_{\bullet}^{\pi} A_{n}$ be the associated graded ring of $A_{n}$ and let $t$ be the principal symbol of $\pi$. Of course, $g r_{\bullet}^{\pi} A_{n}=g r_{\bullet}^{\pi} \hat{A}_{n}$. As we have explained above, $g r_{\bullet}^{\pi} A_{n}=\left(A_{n} / \pi A_{n}\right)[t]$ equals the polynomial ring over $A_{n} / \pi A_{n}$ in the variable $t$. In particular,

$$
\operatorname{gr}_{\bullet}^{\pi} \hat{A}_{n, K}=\left(A_{n} / \pi A_{n}\right)\left[t^{ \pm 1}\right] .
$$

Since $\operatorname{Gr}\left(\hat{A}_{n, K}\right)$ is noetherian, the $\operatorname{ring} A_{n} / \pi A_{n}$ is noetherian, too. So $\operatorname{gr}_{\bullet}^{\pi} A_{n}$ is noetherian. Since $A$ is $\pi$-adically separated, by assumption, so is $A_{n}$. Since $\pi$ is a central and regular element in $A_{n}$, we have the Artin-Rees property for the $\pi$ adic filtration on $A_{n}$ [23, Cor. I.4.4.8]. This implies that the Rees ring associated with the $\pi$-adic filtration of $A_{n}$ is noetherian [23, Thm. II.1.1.5] and this finally allows us to apply the theory of lifted Ore sets as explained in [24. To do this, let $T_{n} \subseteq g r_{\bullet}^{\pi} A_{n}$ be the central and multiplicative subset equal to $\left\{1, t, t^{2}, \ldots\right\}$ and put

$$
S_{n}:=\left\{s \in A_{n}: \sigma(s) \in T\right\} .
$$

Lemma 3.7. The set $S_{n}$ is an Ore set in $A_{n}$. There is a filtration on the localization $S_{n}^{-1} A_{n}$ making $A_{n} \rightarrow S_{n}^{-1} A_{n}$ a filtered homomorphism. The associated graded ring is canonically isomorphic to the localization $T_{n}^{-1}\left(\mathrm{gr}_{\bullet}^{\pi} A_{n}\right)$. The completion homomorphism $S_{n}^{-1} A_{n} \rightarrow \widehat{S_{n}^{-1} A}$ is faithfully flat.

Proof. The statements about the Ore set, the filtration and the graded ring follow from [24, Cor. 2.2/Cor. 2.4]. Note that the filtration on $S_{n}^{-1} A_{n}$ is Zariskian in the sense of [23] and therefore $S_{n}^{-1} A_{n} \rightarrow \widehat{S_{n}^{-1} A_{n}}$ is indeed faithfully flat [23, Thm. II.2.1.2].

Lemma 3.8. In the situation of the preceding lemma, the canonical homomorphism $A_{n} \rightarrow \hat{A}_{n, K}$ extends to an isomorphism of $K$-algebras

$$
\widehat{S_{n}^{-1} A_{n}} \stackrel{\simeq}{\longrightarrow} \hat{A}_{n, K} \text {. }
$$

Proof. The canonical homomorphism $h: A_{n} \rightarrow \hat{A}_{n, K}$ is of course filtered relative to $\pi$-adic filtrations. Moreover, $h\left(1+I_{n}\right)$ consists of units in $\hat{A}_{n}$ which implies $h(s) \in\left(\hat{A}_{n, K}\right)^{\times}$for each $s \in S_{n}$. For any $m$ we denote the homogeneous component of $g r_{\bullet}^{\pi} A_{n}$ of degree $m$ by $g r_{m}^{\pi} A_{n}$, and similarly for the graded rings $g r_{\bullet}^{\pi} \hat{A}$ and $g r^{\pi} \hat{A}_{n, K}$. Given $s \in S_{n}$ with $\sigma(s) \in g r_{m}^{\pi} A_{n}$ we have $\sigma(h(s)) \in g r_{m}^{\pi} \hat{A}_{n, K}$. We have already explained that $A_{n} / \pi A_{n}$ is an integral domain. Hence, the graded $\operatorname{ring} g r_{\bullet}^{\pi} \hat{A}_{n, K}=\left(A_{n} / \pi A_{n}\right)\left[t^{ \pm 1}\right]$ is an integral domain, too, and therefore its principal symbol map is multiplicative. Since $\sigma(1)=1 \in g r_{0} \hat{A}_{n, K}$, we deduce that $\sigma\left(h(s)^{-1}\right) \in g r_{-m} \hat{A}_{n, K}$. The universal property of microlocalization [23, Prop. IV.1.1.3] applied to $h$ therefore yields a filtered homomorphism

$$
\hat{h}: \widehat{S_{n}^{-1} A_{n}} \rightarrow \hat{A}_{n, K}
$$

such that $h=\hat{h} \circ q$ where $q$ equals the canonical map $A_{n} \rightarrow \widehat{S_{n}^{-1} A_{n}}$. We claim that $\hat{h}$ is an isomorphism. Since the filtrations on source and target are exhaustive, separated and complete, it suffices to check that its graded map is an isomorphism 
[23, Cor. I.4.2.5]. However this graded map equals the canonical map between the graded ring of $S_{n}^{-1} A_{n}$ and $g r_{\bullet}^{\pi} \hat{A}_{n, K}=T_{n}^{-1}\left(g r_{\bullet}^{\pi} A_{n}\right)$ which is an isomorphism according to the preceding lemma.

We now turn to the proof of the proposition.

Proof. Consider a (left) ideal $J \subset A_{K}$. Since $A_{K}=A_{n} \otimes_{o_{K}} K \rightarrow \hat{A}_{n, K}$ is flat, the map

$$
\hat{A}_{n, K} \otimes_{A_{K}} J \longrightarrow \hat{A}_{n, K}
$$

is injective for any $n$. The ring $A_{K}$ being noetherian, the $A_{K}$-module $J$ is finitely presented and, hence, so is the $\hat{A}_{K}$-module $\hat{A}_{K} \otimes_{A_{K}} J$. It is therefore a coadmissible module for the Fréchet-Stein algebra $\hat{A}_{K}$ [41, Cor. 3.4] and, consequently, equals the projective limit over the modules $\hat{A}_{n, K} \otimes_{A_{K}} J$. Since the projective limit is leftexact, we thereby obtain from (3.9) the injectivity of the map $\hat{A}_{K} \otimes_{A_{K}} J \rightarrow \hat{A}_{K}$. This establishes the flatness of the map $A_{K} \rightarrow \hat{A}_{K}$.

We turn to faithful flatness. To this end, consider a (left) $A_{K}$-module $M$ and assume $\hat{A}_{K} \otimes_{A_{K}} M=0$. Since $A_{K} \rightarrow \hat{A}_{K}$ is flat, we may assume [5, 3.3.5] that $M$ is a cyclic module on one generator, say $m$. According to the first lemma, the completion homomorphism $S_{n}^{-1} A_{n} \rightarrow \widehat{S_{n}^{-1} A_{n}}$ is faithfully flat. Moreover, $\pi \in S_{n}$, so that $S_{n}^{-1} A_{n}=S_{n}^{-1} A_{K}$. According to the second lemma, we have an isomorphism $\widehat{S_{n}^{-1} A_{n}} \simeq \hat{A}_{n, K}$. We may therefore deduce from

$$
\widehat{S_{n}^{-1} A_{n}} \otimes_{S_{n}^{-1} A_{n}} S_{n}^{-1} M=\hat{A}_{n, K} \otimes_{A_{K}} M=\hat{A}_{n, K} \otimes_{\hat{A}_{K}}\left(\hat{A}_{K} \otimes_{A_{K}} M\right)=0
$$

that $S_{n}^{-1} M=0$; in other words, $M$ is $S_{n}$-torsion for all $n$. Thus, there exists an element $f_{n} \in S_{n}$ with $f_{n} m=0$ for all $n$. However, $S_{n}$ is of the form $\bigcup_{m \geqslant 0} \pi^{m} \cdot(1+$ $I_{n}$ ) where $I_{n}$ denotes the ideal generated by $\pi$ in $A_{n}=\sum_{i \geqslant 0} \pi^{n i} F_{i} A$. Since $F_{0} A$ is $\pi$-adically complete, the elements in $1+\pi F_{0} A$ are units in $F_{0} A$ which allow us to assume that $f_{n}$ is of the form $1+\pi^{n} g_{n}$ with some element $g_{n} \in A$. The limit of the sequence $f_{n} m \in A m$ in the $\pi$-adic topology equals $m$. Thus, $m=0$ and $M=0$. This completes the proof of the proposition.

Corollary 3.10. Let $M$ be a finitely generated $A_{K}$-module and $\hat{M}:=\hat{A}_{K} \otimes_{A_{K}} M$. Then

$$
j_{A_{K}}(M)=j_{\hat{A}_{K}}(\hat{M}) .
$$

Proof. Use Proposition 3.6 and Lemma 2.2 .

We have already explained that the $\operatorname{ring} \operatorname{Gr}\left(\hat{A}_{n, K}\right)$ is a noetherian regular integral domain. Let $d$ denote its (finite) global dimension. Of course, $d$ equals the sum of the global dimension of $F_{0} A / \pi F_{0} A$ and the number $r$ as defined in (2).

Proposition 3.11. The noetherian ring $\hat{A}_{n, K}$ is Auslander regular of global dimension $\leqslant d$.

Proof. The ring $\operatorname{Gr}\left(\hat{A}_{n, K}\right)$ is Auslander regular [23, III.2.4.3] and therefore $A_{n} / \pi A_{n}$ is Auslander regular of global dimension $\leqslant d$ according to [23. II.3.1.4] and 23 , III.2.2.5]. According to [23, III.3.4.6] we obtain that the rings $g r_{\bullet}^{\pi} \hat{A}_{n}=\left(A_{n} / \pi A_{n}\right)[t]$ and $g r^{\pi} \hat{A}_{n, K}=\left(A_{n} / \pi A_{n}\right)\left[t^{ \pm 1}\right]$ are Auslander regular of global dimension $\leqslant d+1$. A second application of [23, II.3.1.4] and [23, III.2.2.5] now yields that the rings $\hat{A}_{n}$ 
and $\hat{A}_{n, K}$ are Auslander regular of global dimension $\leqslant d+1$. On the other hand, $\pi \hat{A}_{n}$ is contained in the Jacobson radical of $\hat{A}_{n}$ according to [23, I.3.3.5] and so $\pi$ annihilates any simple $\hat{A}_{n}$-module. Hence the global dimension of $\hat{A}_{n, K}=\hat{A}_{n}\left[\pi^{-1}\right]$ is even $\leqslant d$ by [26, 7.4.3/7.4.4].

According to the proposition the Fréchet-Stein algebra $\hat{A}_{K}$ verifies the assumption (DIM) as formulated in [41, 8.8]. Consequently, the grade number $j_{\hat{A}_{K}}$ is a well-behaved codimension function on the abelian category of coadmissible modules. This implies the following corollary; cf. [41, Lem. 8.4].

Corollary 3.12. If $M$ is a coadmissible $\hat{A}_{K}$-module and $M_{n}:=\hat{A}_{n, K} \otimes_{\hat{A}_{K}} M$, then

$$
j_{\hat{A}_{K}}(M)=\min _{n} j_{\hat{A}_{n, K}}\left(M_{n}\right) .
$$

We finish with a discussion of examples of algebras $A$ satisfying our requirements. Let $\mathfrak{g}$ be an $R$-Lie algebra which is finite and free as an $R$-module, say of rank $d$. Let $A:=U(\mathfrak{g})$ be its universal enveloping algebra equipped with its usual positive filtration. Then $A$ satisfies all our requirements. Indeed, $A$ is free as an $R$-module, hence $\pi$-adically separated. Moreover, (1) is trivial since $F_{0} A=R$ and the graded ring of $U(\mathfrak{g})$ equals the symmetric algebra of the $R$-module $\mathfrak{g}$ whence (2). Note that $A_{K}=U\left(\mathfrak{g}_{K}\right)$ with the $K$-Lie algebra $\mathfrak{g}_{K}:=\mathfrak{g} \otimes_{R} K$ and that $\hat{A}_{n, K}=\hat{U}\left(\pi^{n} \mathfrak{g}\right)_{K}$, i.e., $\hat{A}_{n, K}$ coincides with the $\pi$-adic completion with subsequent inversion of $\pi$ of the universal enveloping algebra $U\left(\pi^{n} \mathfrak{g}\right)$ of the $R$-Lie algebra $\pi^{n} \mathfrak{g}$ for all $n$. Note that $\operatorname{Gr}\left(\hat{A}_{n, K}\right)$ is isomorphic to the symmetric algebra of the $R / \pi R$-vector space $\mathfrak{g} / \pi \mathfrak{g}$. In particular, the global dimension of $\hat{U}\left(\pi^{n} \mathfrak{g}\right)_{K}$ is in fact equal to $d$ as follows from [3, Prop. 9.1] applied to the augmentation character $\hat{U}\left(\pi^{n} \mathfrak{g}\right)_{K} \rightarrow K$ given by $x=0$ for all $x \in \pi^{n} \mathfrak{g}$. Since $F_{0} A=R$, the Arens-Michael envelope $\hat{A}_{K}$ equals the completion of $U\left(\mathfrak{g}_{K}\right)$ with respect to all submultiplicative seminorms on the abstract $K$-algebra $U\left(\mathfrak{g}_{K}\right)$. This completion was first introduced and studied in [34] and 35. For future reference we restate its faithful flatness property.

Theorem 3.13. The natural homomorphism $U\left(\mathfrak{g}_{K}\right) \rightarrow \hat{U}\left(\mathfrak{g}_{K}\right)$ is faithfully flat.

As a second example we consider a smooth affine integral scheme $X$ of finite type over $R$ whose closed fiber is integral and whose affine algebra $\mathcal{O}(X)$ is $\pi$-adically complete. We assume that the locally free module of differentials $\Omega_{X / R}$ is already free, say of rank $d$. Let $A:=\mathcal{D}(X)$ be the ring of (crystalline) global differential operators on $X$ with its natural filtration. Note that $\mathcal{D}(X)$ coincides with the derivation ring of $\mathcal{O}(X)$ as studied in [26, 15.1]. In particular, $F_{0} A=\mathcal{O}(X)$, the ring of global sections of $X$. Then $A$ satisfies all of our requirements: $A$ is free as an $\mathcal{O}(X)$-module and hence $\pi$-adically separated. Moreover, (1) follows from $F_{0} A=\mathcal{O}(X)$ and our assumptions on $X$. It is well known that the graded ring of $\mathcal{D}(X)$ equals the symmetric algebra of the $\mathcal{O}(X)$-module consisting of the global vector fields on $X$ whence (2).

More generally, the enveloping algebra of a Lie algebroid 31 gives rise to many examples. Let us briefly recall the definition (taken from [1]). Let $R \rightarrow S$ be a ring homomorphism to some commutative ring $S$. A Lie algebroid is a pair $(L, a)$ consisting of an $R$-Lie algebra and $S$-module $L$, together with an $S$-linear $R$-Lie algebra homomorphism $a$ from $L$ to the $R$-linear derivations of $S$, such that $[v, s w]=s[v, w]+a(v)(s) w$ for all $v, w \in L$ and $s \in S$. It is possible to form a unital associative $R$-algebra $U(L)$ called the enveloping algebra of $(L, a)$ which is generated 
as an $R$-algebra by $S$ and $L$ subject to appropriate natural relations. Whenever $L$ is a projective $S$-module, $U(L)$ has a natural positive filtration with associated graded ring the symmetric algebra $\operatorname{Sym}_{S}(L)$. Suppose now that $L$ is already a free $S$-module, say of rank $d$, and that $S$ is $\pi$-adically complete. Then $F_{0} A=S$ and $A:=U(L)$ satisfies all our requirements if and only if $F_{0} A=S$ satisfies (1). Our two first examples above are the special cases $S:=R$ and $(L, a):=(\mathfrak{g}, 0)$, respectively, $S:=\mathcal{O}(X)$ and $(L, a):=\left(\Omega_{X / R}^{\vee}(X), i d\right)$.

\section{From $D(\mathfrak{g}, P)$-modules to $D(G)$-modules}

We consider the locally $L$-analytic groups $P$ and $G$ as well as the maximal compact subgroup $G_{0} \subseteq G$. We let $P_{0}=G_{0} \cap P$ and $\mathfrak{g}=\operatorname{Lie}(G)$. The locally analytic distribution algebras with coefficients in $K$ are denoted by $D(P), D(G), D\left(P_{0}\right)$ and $D\left(G_{0}\right)$. In this section, we will consider a certain functor $\mathcal{F}_{P}^{G}(.)^{\prime}$ from Lie algebra representations of $\mathfrak{g}$ endowed with a compatible locally analytic action of $P$ to locally analytic $G$-representations. This functor, or rather its restriction to certain highest weight categories was introduced and studied in [28. To alleviate notation, we denote the universal enveloping algebra of the base change to $K$ of the $L$-Lie algebra $\mathfrak{g}$ by $U(\mathfrak{g})$.

The group $G$ and its subgroup $P$ act via the adjoint representation on the Lie algebra $\mathfrak{g}$ and hence on $U(\mathfrak{g})$. We denote by

$$
D(\mathfrak{g}, P):=D(P) \otimes_{U(\mathfrak{p})} U(\mathfrak{g})
$$

the corresponding skew-product ring. The skew-multiplication here is induced by

$$
\left(\delta_{p^{\prime}} \otimes x^{\prime}\right) \cdot\left(\delta_{p} \otimes x\right)=\delta_{p^{\prime} p} \otimes \delta_{p^{-1}}\left(x^{\prime}\right) x
$$

for $p, p^{\prime} \in P$ and $x, x^{\prime} \in U(\mathfrak{g})$. Similarly, we denote by $D\left(\mathfrak{g}, P_{0}\right)$ the skew-product $\operatorname{ring} D\left(P_{0}\right) \otimes_{U(\mathfrak{p})} U(\mathfrak{g})$.

Lemma 4.1. The natural linear map $D(\mathfrak{g}, P) \rightarrow D(G)$ is an injective ring homomorphism with image equal to the subring of $D(G)$ generated by $D(P)$ and $U(\mathfrak{g})$.

Proof. Let $U_{P}^{-}$be the group of points of the opposite unipotent radical of $P$ and let $\mathfrak{u}_{\mathfrak{p}}^{-}$be its Lie-algebra. In particular, $\mathfrak{g}=\mathfrak{p} \oplus \mathfrak{u}_{\mathfrak{p}}^{-}$. The multiplication map $P \times U_{P}^{-} \rightarrow$ $G$ is injective and induces an injective homomorphism $D\left(P \times U_{P}^{-}\right) \rightarrow D(G)$. The linear map appearing in the lemma is injective being the composite of the injective linear maps

$$
D(\mathfrak{g}, P)=D(P) \otimes_{K} U\left(\mathfrak{u}_{\mathfrak{p}}^{-}\right) \longrightarrow D(P) \otimes_{K} D\left(U_{P}^{-}\right) \longrightarrow D\left(P \times U_{P}^{-}\right) \longrightarrow D(G) .
$$

The remaining assertions are clear.

An obvious variant of the above proof for the group $G_{0}$ shows that the natural linear map $D\left(\mathfrak{g}, P_{0}\right) \rightarrow D\left(G_{0}\right)$ is an injective ring homomorphism with image equal to the subring of $D\left(G_{0}\right)$ generated by $D\left(P_{0}\right)$ and $U(\mathfrak{g})$.

Lemma 4.2. One has

$$
D(G)=D\left(G_{0}\right) \otimes_{D\left(\mathfrak{g}, P_{0}\right)} D(\mathfrak{g}, P)
$$

as bimodules. In particular,

$$
D(G) \otimes_{D(\mathfrak{g}, P)} M=D\left(G_{0}\right) \otimes_{D\left(\mathfrak{g}, P_{0}\right)} M
$$

for any $D(\mathfrak{g}, P)$-module $M$. 
Proof. The bimodule map equal to the composite

$$
D\left(G_{0}\right) \otimes_{D\left(P_{0}\right)} D(P) \longrightarrow D\left(G_{0}\right) \otimes_{D\left(\mathfrak{g}, P_{0}\right)} D(\mathfrak{g}, P) \rightarrow D(G)
$$

is an isomorphism according to [42, Lem. 6.1]. Since the first map is surjective, both individual maps are isomorphisms as well. The second statement is clear.

We consider the functor

$$
M \mapsto \mathcal{F}_{P}^{G}(M)^{\prime}:=D(G) \otimes_{D(\mathfrak{g}, P)} M
$$

from $D(\mathfrak{g}, P)$-modules to $D(G)$-modules. Here, we follow the notation of [28], compare in particular Proposition 3.7 in loc. cit. If the parabolic subgroup $P$ is clear from the context, we will occasionally abbreviate

$$
\mathbf{M}:=\mathcal{F}_{P}^{G}(M)^{\prime} .
$$

Lemma 4.3. If $M$ is finitely generated as $U(\mathfrak{g})$-module, then $\mathbf{M}$ is coadmissible.

Proof. As a $D\left(G_{0}\right)$-module we have $\mathbf{M}=D\left(G_{0}\right) \otimes_{D\left(\mathfrak{g}, P_{0}\right)} M$ according to the preceding lemma. The group $P_{0}$ is topologically finitely generated. Let $p_{1}, \ldots, p_{r}$ be a set of topological generators and let $m_{1}, \ldots, m_{s}$ be a set of generators for the $U(\mathfrak{g})$ module $M$. Since $U(\mathfrak{g})$ is noetherian, the $D\left(G_{0}\right)$-module $D\left(G_{0}\right) \otimes_{U(\mathfrak{g})} M$ is finitely presented and hence coadmissible. Consider its submodule $N$ finitely generated by the elements $\delta_{p_{i}} \otimes m_{j}-1 \otimes \delta_{p_{i}} m_{j}$. Then $N$ is coadmissible and it suffices to see that $N$ equals the kernel of the natural surjection $D\left(G_{0}\right) \otimes_{U(\mathfrak{g})} M \rightarrow \mathbf{M}$, i.e., that $N$ contains all elements of the form $\delta \otimes m-1 \otimes \delta m$ where $\delta \in D\left(P_{0}\right)$ and $m \in M$. To this end, observe that $M$ equals a countable union of vector subspaces of finite dimension. We give $M$ the finest locally convex topology and let $W:=D\left(G_{0}\right) \otimes_{K} M$ have its projective tensor product topology, e.g., [37, §17B]. Then $W$ satisfies the assumptions of [37, Prop. 8.8]. Indeed, $M$ is a finitely generated module over $U(\mathfrak{g})$ and hence equals a countable locally convex inductive limit over finite-dimensional subspaces $M_{n}$, each $W_{n}:=D\left(G_{0}\right) \otimes_{K} M_{n}$ is a Fréchet space which continuously embeds into $W$ and $W=\bigcup_{n} W_{n}$. Hence, the surjective continuous linear map $W \rightarrow D\left(G_{0}\right) \otimes_{U(\mathfrak{g})} M$ is open. In other words, the canonical topology on the coadmissible module $D\left(G_{0}\right) \otimes_{U(\mathfrak{g})} M$ equals the quotient topology of $W$ by a suitable closed subspace. Hence, if $\delta_{n} \rightarrow \delta$ is a convergent sequence in $D\left(P_{0}\right)$, then for any $m \in M$,

$$
\left(\delta_{n} \otimes m-1 \otimes \delta_{n} m\right) \rightarrow(\delta \otimes m-1 \otimes \delta m)
$$

is a convergent sequence in the coadmissible module $D\left(G_{0}\right) \otimes_{U(\mathfrak{g})} M$. Since $N$ is closed in $D\left(G_{0}\right) \otimes_{U(\mathfrak{g})} M$, we are therefore reduced to show that $\delta_{n} \otimes m-1 \otimes \delta_{n} m \in N$ for all $n$. Since the abstract group ring $K\left[P_{0}\right]$ is dense in $D\left(P_{0}\right)$ according to [40, Lem. 3.1] we may assume $\delta_{n} \in K\left[P_{0}\right]$. Since our assertion is linear in $\delta_{n}$, we may further assume that $\delta_{n}$ lies in the image of $P_{0}$. The assertion follows in this case from the fact that the $p_{i}$ topologically generate the group $P_{0}$.

We now start a more detailed analysis of the module $\mathbf{M}$ closely following the discussion in [28, 5.5]. We put

$$
\kappa= \begin{cases}1, & p>2, \\ 2, & p=2 .\end{cases}
$$


In the following, let $r$ always denote a real number in $(0,1) \cap p^{\mathbb{Q}}$ with the property:

(4.4) there is $m \in \mathbb{Z}_{\geqslant 0}$ such that $s=r^{p^{m}}$ satisfies $\frac{1}{p}<s$ and $s^{\kappa}<p^{-1 /(p-1)}$.

For such numbers $r$ we let $D_{r}\left(G_{0}\right)$ and $D_{r}\left(P_{0}\right)$ be the Banach algebras appearing in loc. cit. Let us briefly sketch their construction. One chooses suitable uniform pro- $p$ groups $H \subset G_{0}$ and $H^{+}:=H \cap P_{0}$ such that $H$ is open normal in $G_{0}$. The distribution algebras of $H$ and $H^{+}$admit canonical $r$-norms coming from the canonical $p$-valuation on the group [41. The rings $D\left(G_{0}\right)$ resp. $D\left(P_{0}\right)$ are finite free ring extensions over $D(H)$ resp. $D\left(H^{+}\right)$and carry the corresponding maximum norms. The rings $D_{r}\left(G_{0}\right)$ resp. $D_{r}\left(P_{0}\right)$ are the associated completions. They define the Fréchet-Stein structure of $D\left(G_{0}\right)$ resp. $D\left(P_{0}\right)$. Let $U_{r}(\mathfrak{g})$ and $U_{r}(\mathfrak{p})$ be the topological closure of $U(\mathfrak{g})$ in $D_{r}\left(G_{0}\right)$ and $U(\mathfrak{p})$ in $D_{r}\left(P_{0}\right)$ respectively. Put

$$
D_{r}\left(\mathfrak{g}, P_{0}\right):=D_{r}\left(P_{0}\right) \otimes_{U_{r}(\mathfrak{p})} U_{r}(\mathfrak{g}) .
$$

An argument completely analogous to Lemma 4.1 shows that the natural linear map $D_{r}\left(\mathfrak{g}, P_{0}\right) \rightarrow D_{r}\left(G_{0}\right)$ is an injective ring homomorphism with image equal to the subring of $D_{r}\left(G_{0}\right)$ generated by $D_{r}\left(P_{0}\right)$ and $U_{r}(\mathfrak{g})$. If $H P_{0}$ denotes the subgroup of $G_{0}$ generated by $H$ and $P_{0}$, the intersection

$$
P_{0, r}:=H P_{0} \cap D_{r}\left(\mathfrak{g}, P_{0}\right)
$$

is thus well defined.

Lemma 4.5. The set $P_{0, r}$ is an open normal subgroup of $H P_{0}$. One has

$$
D_{r}\left(G_{0}\right)=\bigoplus_{g \in G_{0} / P_{0, r}} \delta_{g} D_{r}\left(\mathfrak{g}, P_{0}\right)
$$

Proof. This follows from [28, 5.6].

We let

$$
M_{r}:=U_{r}(\mathfrak{g}) \otimes_{U(\mathfrak{g})} M, \quad \mathbf{M}_{r}:=D_{r}\left(G_{0}\right) \otimes_{D\left(G_{0}\right)} \mathbf{M}=D_{r}\left(G_{0}\right) \otimes_{D\left(\mathfrak{g}, P_{0}\right)} M .
$$

For $g \in G$ we denote by $\operatorname{Ad}(g)$ the automorphism of $U(\mathfrak{g})$ (or $U_{r}(\mathfrak{g})$ ) induced by the left conjugation action $h \mapsto g h g^{-1}$ of $g$ on $G$. We note that the group $P_{0}$ acts on $M_{r}$ via $p .(\mathfrak{x} \otimes m):=(\operatorname{Ad}(p)(\mathfrak{x})) \otimes p m$.

Lemma 4.6. The natural map

$$
M_{r} \stackrel{\simeq}{\longrightarrow} D_{r}\left(\mathfrak{g}, P_{0}\right) \otimes_{D\left(\mathfrak{g}, P_{0}\right)} M
$$

induced from the map $U_{r}(\mathfrak{g}) \rightarrow D_{r}\left(\mathfrak{g}, P_{0}\right), \mathfrak{x} \mapsto 1 \otimes \mathfrak{x}$ is bijective.

Proof. The ring $D_{r}\left(P_{0}\right)$ is a finite and free module over $U_{r}(\mathfrak{p})$ on a basis given by distributions $\delta_{p}$ with $p \in P_{0}$. The $\left(P_{0}, U_{r}(\mathfrak{g})\right)$-module structure on $M_{r}$ therefore extends to a module structure over the ring $D_{r}\left(\mathfrak{g}, P_{0}\right)$. The resulting map $D_{r}\left(\mathfrak{g}, P_{0}\right) \otimes_{D\left(\mathfrak{g}, P_{0}\right)} M \rightarrow M_{r}$ provides an inverse for the map in question.

Using the two lemmas we can derive the following decomposition of $\mathbf{M}_{r}$ as $U_{r}(\mathfrak{g})$ module,

$$
\mathbf{M}_{r}=D_{r}\left(G_{0}\right) \otimes_{D\left(\mathfrak{g}, P_{0}\right)} M=D_{r}\left(G_{0}\right) \otimes_{D_{r}\left(\mathfrak{g}, P_{0}\right)} M_{r} \simeq \bigoplus_{g \in G_{0} / P_{0, r}} \delta_{g} \star M_{r},
$$

where $\delta_{g} \star M_{r}$ denotes the twist of the $U_{r}(\mathfrak{g})$-module $M_{r}$ with the automorphism $\operatorname{Ad}(g)$ in the sense of Lemma 2.1. 
Proposition 4.8. On the abelian category of $D(\mathfrak{g}, P)$-modules which are finitely generated as $U(\mathfrak{g})$-modules, the correspondence $M \mapsto \mathbf{M}$ constitutes an exact and faithful functor with trivial kernel (i.e., $\mathbf{M}=0$ implies $M=0$ ).

Proof. By results of Frommer and Kohlhaase (in the form of [33, 2.3]) the projective limit $\hat{U}(\mathfrak{g})=\lim _{r} U_{r}(\mathfrak{g})$ is Fréchet-Stein and equals the Arens-Michael envelope (also called hyperenveloping algebra in loc. cit.) of $U(\mathfrak{g})$. By [14, 1.2.7] the projective system $U_{r}(\mathfrak{g})$ and the projective system used in Theorem 3.13 are therefore equivalent, but we will not need this. If $M \neq 0$, then

$$
\hat{M}:=\hat{U}(\mathfrak{g}) \otimes_{U(\mathfrak{g})} M \neq 0
$$

according to Theorem 3.13. Moreover, since $M$ is finitely generated, the $\hat{U}(\mathfrak{g})$ module $\hat{M}$ is finitely presented and hence coadmissible. Thus, $\hat{M}=\lim _{r} M_{r}$ which implies $M_{r} \neq 0$ for a cofinal family of values of $r$. According to (4.7), $\mathbf{M}_{r} \neq 0$ for these $r$. Since $\mathbf{M}$ is coadmissible (4.3), this implies $\mathbf{M} \neq 0$. Moreover, a sequence of coadmissible $D\left(G_{0}\right)$-modules is exact if and only if this is true after base extension to $D_{r}\left(G_{0}\right)$ for a cofinal family of values of $r$. The decomposition (4.7) is natural in $M$. Since the functor $M \mapsto M_{r}$ is exact [41, Rem. 3.2], so is the functor $M \mapsto \mathbf{M}$. The faithfulness is now a formal consequence.

We compute a class of examples related to locally analytic parabolic induction. Recall the Levi decompositions $P=L_{P} \cdot U_{P}$ and $\mathfrak{p}=\mathfrak{l}_{P} \oplus \mathfrak{u}_{\mathfrak{p}}$. Let $V$ be a locally analytic $L_{P}$-representation on a finite-dimensional $K$-vector space. We set $\mathfrak{u}_{\mathfrak{p}} V=0$ and consider $V$ a $U(\mathfrak{p})$-module. The induced $U(\mathfrak{g})$-module

$$
M(V):=U(\mathfrak{g}) \otimes_{U(\mathfrak{p})} V
$$

is then naturally a $D(\mathfrak{g}, P)$-module which is finitely generated over $U(\mathfrak{g})$. Indeed, we have the diagonal action of $L_{P}$ on the tensor product $M(V)$ where $L_{P}$ acts on the factor $U(\mathfrak{g})$ via the adjoint action. It extends to a $D\left(L_{P}\right)$-action and it suffices therefore to check that the $\mathfrak{u}_{\mathfrak{p}}$-action extends compatibly to $D\left(U_{P}\right)$. However, the action of the Lie algebra $\mathfrak{u}_{\mathfrak{p}}$ even integrates uniquely to an algebraic action of $U_{P}$ on $M(V)$ as follows. Given an element $u=\exp (\mathfrak{x}) \in \mathbf{U}_{\mathbf{P}}(\bar{K})$, where $\bar{K}$ denotes an algebraic closure of $K$, we define $\rho(u):=\sum_{n \geqslant 0} \frac{\rho(\mathfrak{x})^{n}}{n !}$, where $\rho(\mathfrak{x})^{n}=0$ for $n \gg 0$. The representations of $L_{P}$ and $U_{P}$ are compatible in the sense that $h \circ \rho(u) \circ h^{-1}=\rho(\operatorname{Ad}(h)(u))$, for $h \in L_{P}, u \in U_{P}$. Hence, $M(V)$ is a $D(P)$-module and then even a $D(\mathfrak{g}, P)$-module as claimed.

Proposition 4.9. The map $V \rightarrow M(V), v \mapsto 1 \otimes v$ induces an isomorphism of $D(G)$-modules

$$
D(G) \otimes_{D(P)} V \stackrel{\simeq}{\longrightarrow} \mathcal{F}_{P}^{G}(M(V))^{\prime}
$$

Proof. The map

$$
\mathcal{F}_{P}^{G}(M(V))^{\prime} \rightarrow D(G) \otimes_{D(P)} V, \delta \otimes(x \otimes v) \mapsto(\delta x) \otimes v
$$

for $\delta \in D(G), x \in U(\mathfrak{g}), v \in V$ is well defined and provides a two-sided inverse.

Remark. The module $D(G) \otimes_{D(P)} V$ is dual to the locally analytic parabolic induction $\operatorname{Ind}_{P}^{G}\left(V^{\prime}\right)$. 
In the following we will investigate the behavior of the functor $\mathcal{F}_{P}^{G}(.)^{\prime}$ in terms of dimensions. To this end, recall that the ring $U(\mathfrak{g})$ is a noetherian Auslander regular ring of global dimension $d:=\operatorname{dim}_{L} \mathfrak{g}$. For a finitely generated $U(\mathfrak{g})$-module $M$ we therefore have its canonical dimension $\operatorname{dim}_{U(\mathfrak{g})} M:=d-j_{U(\mathfrak{g})}(M)$; cf. section 2 ,

Remark. Traditionally, dimension theory over the ring $U(\mathfrak{g})$ is developed using the so-called Gelfand-Kirillov dimension; cf. [17. However, it follows from [22, Remark 5.8 (3)] together with [26. Prop. 8.1.15 (iii)] that for finitely generated $U(\mathfrak{g})$-modules, Gelfand-Kirillov dimension coincides with canonical dimension.

On the other hand, for any compact open subgroup $H \subseteq G$ and a coadmissible $D(H)$-module $M$, we define

$$
\operatorname{dim}_{D(H)} M:=d-j_{D(H)}(M) .
$$

If $D(H)=\lim _{r} D_{r}(H)$ is a Fréchet-Stein structure for $D(H)$ and $M_{r}:=D_{r}(H)$ $\otimes_{D(H)} M$, then

$$
\operatorname{dim}_{D(H)}(M)=\sup _{r} \operatorname{dim}_{D_{r}(H)}\left(M_{r}\right)
$$

according to [41, §8]. Moreover, if $M$ is even a $D(G)$-module, then, according to [41] and [32, the number $\operatorname{dim}_{D(H)} M$ is independent of the choice of $H$. In this case, we denote it by $\operatorname{dim}_{D(G)} M$, or simply $\operatorname{dim} M$, if no confusion can arise, and call it the canonical dimension of the coadmissible $D(G)$-module $M$.

We shall also need the Arens-Michael envelope $\hat{U}(\mathfrak{g})$ of $\mathfrak{g}$ as introduced in the preceding section. Recall that this is a Fréchet-Stein algebra equal to the completion of $U(\mathfrak{g})$ with respect to all submultiplicative seminorms on $U(\mathfrak{g})$. As such, it comes with a natural completion homomorphism $U(\mathfrak{g}) \rightarrow \hat{U}(\mathfrak{g})$ which is faithfully flat; cf. Theorem 3.13 .

Theorem 4.11. If $M$ is a $D(\mathfrak{g}, P)$-module which is finitely generated as $U(\mathfrak{g})$ module, then

$$
\operatorname{dim}_{D(G)} \mathbf{M}=\operatorname{dim}_{U(\mathfrak{g})} M .
$$

Proof. It suffices to prove $j_{D\left(G_{0}\right)}(\mathbf{M})=j_{U(\mathfrak{g})}(M)$. The left-hand side of this identity equals $\min _{r} j_{D_{r}\left(G_{0}\right)}\left(\mathbf{M}_{r}\right)$ according to (4.10). According to [32, 6.3] (compare also [28, (5.5.7)]) the ring $D_{r}\left(G_{0}\right)$ is a finite free $U_{r}(\mathfrak{g})$-module on a basis which consists of units satisfying the assumptions of [41, Lem. 8.8]. Hence, $j_{D_{r}\left(G_{0}\right)}\left(\mathbf{M}_{r}\right)=j_{U_{r}(\mathfrak{g})}\left(\mathbf{M}_{r}\right)$ for all $r$. By (4.7) together with Lemma 2.1, we have

$$
j_{U_{r}(\mathfrak{g})}\left(\mathbf{M}_{r}\right)=\max _{g \in G_{0} / P_{0, r}} j_{U_{r}(\mathfrak{g})}\left(\delta_{g} \star M_{r}\right)=j_{U_{r}(\mathfrak{g})} M_{r}
$$

So it remains to show that $j_{U(\mathfrak{g})}(M)=\min _{r} j_{U_{r}(\mathfrak{g})}\left(M_{r}\right)$. Since $\hat{M}:=\hat{U}(\mathfrak{g}) \otimes_{U(\mathfrak{g})}$ $M$ is coadmissible, we have

$$
j_{U(\mathfrak{g})}(M)=j_{\hat{U}(\mathfrak{g})}(\hat{M})=\min _{r} j_{U_{r}(\mathfrak{g})}\left(M_{r}\right)
$$

according to Corollary 3.10 and Corollary 3.12 .

Combining the theorem with [17, Lem. 8.9] gives the dimension of parabolically induced representations.

Corollary 4.12. One has $\operatorname{dim} \mathcal{F}_{P}^{G}(M(V))^{\prime}=\operatorname{dim}_{L}(\mathfrak{g} / \mathfrak{p})$ where $\operatorname{dim}_{L}$ denotes vector space dimension. 


\section{Highest Weight modules And dimension}

In this section we explain the relation to the parabolic BGG-categories for the pair $\mathfrak{p} \subseteq \mathfrak{g}$ appearing in 28 and compute the dimensions of certain irreducible $G$-representations occurring in the image of the functor $\mathcal{F}_{P}^{G}$. As in the previous section, we make the general convention that, when dealing with universal enveloping algebras, we write $U(\mathfrak{g}), U(\mathfrak{p})$, etc. to denote the corresponding universal enveloping algebras after base change to $K$, i.e., what is precisely $U\left(\mathfrak{g}_{K}\right), U\left(\mathfrak{p}_{K}\right)$ and so on.

5.1. The category $\mathcal{O}$ and its parabolic variants $\mathcal{O}^{\mathfrak{p}}$. We let $\mathcal{O}$ be the full subcategory of all finitely generated $U(\mathfrak{g})$-modules $M$ which satisfy the properties:

(1) $M$ decomposes as a direct sum of one-dimensional $\mathfrak{t}_{K}$-representations;

(2) for every $m \in M$, the subspace $U(\mathfrak{b}) \cdot m \subset M$ is finite-dimensional over $K$. By (1), we may write any object $M$ in $\mathcal{O}$ as a direct sum

$$
M=\bigoplus_{\lambda \in t_{K}^{*}} M_{\lambda}
$$

where $M_{\lambda}=\left\{m \in M \mid \forall \mathfrak{x} \in \mathfrak{t}_{K}: \mathfrak{x} \cdot m=\lambda(\mathfrak{x}) m\right\}$ is the $\lambda$-eigenspace attached to $\lambda \in \mathfrak{t}_{K}^{*}=\operatorname{Hom}_{K}\left(\mathfrak{t}_{K}, K\right)$. Let $X^{*}(\mathbf{T})=\operatorname{Hom}\left(\mathbf{T}, \mathbb{G}_{m}\right)$ be the group of characters of the torus $\mathbf{T}$ which we consider via the derivative as a subgroup of $\mathbf{t}_{K}^{*}$.

We let $\mathcal{O}_{\text {alg }}$ be the full subcategory of $\mathcal{O}$ consisting of objects $M \in \mathcal{O}$ where the $\mathfrak{t}_{K}$-module structure on every $M_{\lambda}$ lifts to an algebraic action of $\mathbf{T}$.

Example 5.2. For $\lambda \in \mathfrak{t}_{K}^{*}$, let $K_{\lambda}=K$ be the 1-dimensional $\mathfrak{t}_{K}$-module where the action is given by $\lambda$. Then $K_{\lambda}$ extends uniquely to a $\mathfrak{b}_{K}$-module. Let

$$
M(\lambda)=U(\mathfrak{g}) \otimes_{U(\mathfrak{b})} K_{\lambda} \in \mathcal{O}
$$

be the corresponding Verma module. Denote by $L(\lambda) \in \mathcal{O}$ its simple quotient. Suppose the character $\lambda$ integrates to a locally analytic character of $T$. As we have explained before Proposition 4.9] the module $M(\lambda)$ is then a $D(\mathfrak{g}, B)$-module finitely generated over $U(\mathfrak{g})$ and the same holds true for $L(\lambda)$. In this situation, $M(\lambda)$ resp. $L(\lambda)$ is an object of $\mathcal{O}_{\text {alg }}$ if and only if $\lambda \in X^{*}(\mathbf{T})$.

We shall also need the parabolic versions of the above categories. We define $\mathcal{O}^{\mathfrak{p}}$ to be the category of finitely generated $U(\mathfrak{g})$-modules $M$ satisfying the properties:

(1) viewed as a $\mathfrak{l}_{\mathfrak{p}, K}$-module, $M$ is the direct sum of finite-dimensional simple modules;

(2) the action of $\mathfrak{u}_{\mathfrak{p}, K}$ on $M$ is locally finite.

Clearly, the category $\mathcal{O}^{\mathfrak{p}}$ is a full subcategory of $\mathcal{O}$. If $Q$ is a standard parabolic subgroup with $Q \supset P$, then $\mathcal{O}^{\mathfrak{q}} \subset \mathcal{O}^{\mathfrak{p}}$. For $\mathfrak{p}=\mathfrak{g}$, the category $\mathcal{O}^{\mathfrak{g}}$ consists of all finite-dimensional semisimple $U(\mathfrak{g})$-modules whereas for $\mathfrak{p}=\mathfrak{b}$, we have $\mathcal{O}^{\mathfrak{b}}=\mathcal{O}$. Let $\operatorname{Irr}\left(\mathfrak{l}_{\mathfrak{p}, K}\right)^{\mathrm{fd}}$ be the set of isomorphism classes of finite-dimensional irreducible $\mathfrak{l}_{\mathfrak{p}, K}$-modules. By (1), any object in $\mathcal{O}^{\mathfrak{p}}$ has a decomposition into $\mathfrak{l}_{\mathfrak{p}, K}$-modules

$$
M=\bigoplus_{\mathfrak{a} \in \operatorname{Irr}\left(\mathfrak{l}_{\mathfrak{p}, K}\right)^{\mathrm{fd}}} M_{\mathfrak{a}}
$$


where $M_{\mathfrak{a}} \subset M$ is the a-isotypic part of the representation $\mathfrak{a}$. We let $\mathcal{O}_{\text {alg }}^{\mathfrak{p}}$ be the full subcategory of $\mathcal{O}^{\mathfrak{p}}$ consisting of objects $M$ of $\mathcal{O}^{\mathfrak{p}}$ with the following property: if $M_{\mathfrak{a}} \neq 0$ (with the notation as in (5.3) ), then $\mathfrak{a}$ is the Lie algebra representation induced by a finite-dimensional algebraic $\mathbf{L}_{\mathbf{P}, K}$-representation, where $\mathbf{L}_{\mathbf{P}, K}=\mathbf{L}_{\mathbf{P}} \times_{\operatorname{Spec}(L)} \operatorname{Spec}(K)$ (note that $\mathfrak{l}_{\mathfrak{p}, K}=\operatorname{Lie}\left(\mathbf{L}_{\mathbf{P}, K}\right)$ ). Again, the category $\mathcal{O}_{\text {alg }}^{\mathfrak{g}}$ is contained in $\mathcal{O}_{\text {alg }}$ and contains all finite-dimensional $\mathfrak{g}_{K}$-modules which are induced by algebraic G-modules. If $M$ is an object of $\mathcal{O}^{\mathfrak{p}}$, then $M$ is in $\mathcal{O}_{\text {alg }}^{\mathfrak{p}}$ if and only it is in $\mathcal{O}_{\text {alg }}$; cf. [28, Lem. 2.8].

Example 5.4. Let $\Delta$ be the set of simple roots of $\mathbf{G}$ with respect to $\mathbf{T} \subset \mathbf{B}$. Let $\lambda \in \mathfrak{t}_{K}^{*}$ and set $I=\left\{\alpha \in \Delta \mid\left\langle\lambda, \alpha^{v}\right\rangle \in \mathbb{Z}_{\geqslant 0}\right\}$. We let $\mathbf{P}=\mathbf{P}_{I}$ is the standard parabolic subgroup of $\mathbf{G}$ attached to $I$. Then $\lambda$ is dominant with respect to the reductive Lie algebra $\mathfrak{l}_{\mathfrak{p}}=\operatorname{Lie}\left(\mathbf{L}_{\mathbf{P}}\right)$. Denote by $V_{I}(\lambda)$ the corresponding irreducible finite-dimensional $\mathfrak{l}_{\mathfrak{p}}$-representation and consider the generalized Verma module (in the sense of Lepowsky [21])

$$
M_{I}(\lambda)=U(\mathfrak{g}) \otimes_{U\left(\mathfrak{p}_{I}\right)} V_{I}(\lambda) .
$$

There is a surjective map $M(\lambda) \rightarrow M_{I}(\lambda)$, where the kernel is given by the image of $\bigoplus_{\alpha \in I} M\left(s_{\alpha} \cdot \lambda\right) \rightarrow M(\lambda)$. Now suppose the $\mathfrak{l}_{\mathfrak{p}}$-representation on $V_{I}(\lambda)$ integrates to a locally analytic $L_{P}$-representation. As we have explained before Proposition 4.9, the module $M_{I}(\lambda)$ is then a $D(\mathfrak{g}, P)$-module and finitely generated over $U(\mathfrak{g})$. In this situation, $M_{I}(\lambda)$ is an object of $\mathcal{O}_{\text {alg }}^{\mathfrak{p}}$ if and only if the $\mathfrak{l}_{\mathfrak{p}}$-action on $V_{I}(\lambda)$ integrates to an algebraic $L_{P}$-action. This happens if and only if $\lambda \in X(\mathbf{T})$. In this case, $L(\lambda)$ is an object of $\mathcal{O}_{\text {alg }}^{\mathfrak{p}}$ [16, sec. 9.4].

Let $M$ be an object of $\mathcal{O}_{\text {alg }}^{\mathfrak{p}}$ as above. Then $M$ is the union of finite-dimensional $\mathfrak{p}_{K}$-modules. Denote by $X$ one of these finite-dimensional submodules. Then $X$ lifts uniquely to an algebraic $\mathbf{P}_{K}$-representation [28, Cor. 3.6]. Let us sketch the argument. The $U(\mathfrak{p})$-module $X$, considered as a $U\left(\mathfrak{l}_{\mathfrak{p}}\right)$-module, decomposes into a direct sum of isotypic modules $X_{\mathfrak{a}}$ and each module $X_{\mathfrak{a}}$ lifts uniquely to an algebraic representation of $\mathbf{L}_{\mathbf{P}, K}$. The action of the Lie algebra $\mathfrak{u}_{\mathfrak{p}, K}$ integrates uniquely to an algebraic action of $\mathbf{U}_{\mathbf{P}}$ on $X$ in the manner we have explained before Proposition 4.9. This shows that $X$ is uniquely endowed with an algebraic representation of $\mathbf{P}_{K}$. Consequently, there is a unique $D(\mathfrak{g}, P)$-module structure on $M$ that extends its $U(\mathfrak{g})$-module structure and such that the action of $U(\mathfrak{p})$, as a subring of $U(\mathfrak{g})$, coincides with the action of $U(\mathfrak{p})$ as a subring of $D(P)$. Moreover, any morphism $M_{1} \rightarrow M_{2}$ in $\mathcal{O}_{\text {alg }}^{\mathfrak{p}}$ is automatically a homomorphism of $D(\mathfrak{g}, P)$-modules. In other words, we have a fully faithful embedding of categories

$$
\mathcal{O}_{\text {alg }}^{\mathfrak{p}} \hookrightarrow \quad \text { category of all } D(\mathfrak{g}, P) \text {-modules, finitely generated over } U(\mathfrak{g}) \text {. }
$$

We now explain how one may compute the dimensions of the irreducible $G$ representations that occur in the image of $\mathcal{O}_{\text {alg }}^{\mathfrak{p}}$ via the functor $\mathcal{F}_{P}^{G}$. Let $\lambda \in \mathfrak{t}_{K}^{*}$ be the differential of a locally analytic character of $T$ and let $P=P_{I}$ be adapted to $\lambda$ in the sense of Example 5.4. Consider the simple quotient $L(\lambda)$ and the coadmissible $D(G)$-module

$$
\mathbf{L}_{I}(\lambda):=\mathcal{F}_{P}^{G}(L(\lambda))^{\prime} .
$$

We have $\operatorname{dim} \mathbf{L}_{I}(\lambda)=\operatorname{dim} L(\lambda)$ by Theorem 4.11. Moreover, if $\lambda \in X^{*}(\mathbf{T})$, then $L(\lambda)$ is an object of $\mathcal{O}_{\text {alg }}^{\mathfrak{p}}$ and the $D(G)$-module $\mathbf{L}_{I}(\lambda)$ is simple (at least if $p \geqslant 5$; cf. [28, Thm. 5.3]). We therefore briefly recall the classical relation of $\operatorname{dim} L(\lambda)$ to 
the so-called Goldie rank polynomials. For this, we need to introduce some extra notation following [17, 2.7]. We let $X^{*}(\mathbf{T}) \subseteq \Lambda$ be the integral weight lattice and let $\Lambda^{+}$and $\Lambda^{++}$be the subsets of dominant resp. strictly dominant weights. For simplicity we assume $\lambda \in \Lambda$. Recall that the isomorphism classes of the $L(\mu), \mu \in \mathfrak{t}^{*}$ as well as the isomorphism classes of the $M(\mu), \mu \in \mathfrak{t}^{*}$ form two different $\mathbb{Z}$-bases of the Grothendieck group of the abelian category $\mathcal{O}$; cf. [17, 4.5]. In particular, for any $\mu \in \mathfrak{t}^{*}$,

$$
[L(\mu)]=\sum_{\mu^{\prime} \in \mathbf{t}^{*}}\left(L(\mu): M\left(\mu^{\prime}\right)\right)\left[M\left(\mu^{\prime}\right)\right]
$$

for some uniquely determined coefficients $\left(L(\mu): M\left(\mu^{\prime}\right)\right) \in \mathbb{Z}$. For any $\mu \in \Lambda^{++}$, the number

$$
a_{\Lambda}\left(w, w^{\prime}\right):=\left(L(w \cdot \mu): M\left(w^{\prime} \cdot \mu\right)\right)
$$

for $w, w^{\prime} \in W$ is independent of the choice of $\mu$, cf. [17, 4.14]. Here, - denotes the dot action of $W$ on $X^{*}(\mathbf{T})_{\mathbb{Q}}$, i.e., $w \cdot \mu=w(\mu+\rho)-\rho$ where $\rho$ equals half the sum over the positive roots $\Phi^{+}$. We fix once and for all an element $t \in \mathfrak{t}$ such that $\alpha(t)=1$ for all $\alpha \in \Delta$. For fixed $w \in W$ we let $m=m_{w} \in \mathbb{N}_{\geqslant 0}$ be minimal such that

$$
\tilde{f}_{w}^{\Lambda}:=\frac{1}{m !} \sum_{w^{\prime} \in W} a_{\Lambda}\left(w, w^{\prime}\right)\left(w^{\prime-1}(t)\right)^{m} \in \operatorname{Sym}^{m}(\mathfrak{t})
$$

is nonzero; cf. [17, 9.13]. The number $m_{w}$ does not depend on the particular choice of $t \in \mathfrak{t}^{*}$. In fact, different choices of $t$ lead to polynomials that differ by a scalar in $L^{\times}$; cf. [17, 14.7]. The polynomial $\tilde{f}_{w}^{\Lambda}$ is, up to scaling, the so-called Goldie rank polynomial of $w \in W$.

Remark. The polynomials $\tilde{f}_{w}^{\Lambda}$ and their generalizations to arbitrary cosets $\mathfrak{t}^{*} / \Lambda$ were introduced and studied by Joseph 19 and build a bridge between primitive ideals of $U(\mathfrak{g})$, nilpotent adjoint orbits and the representation theory of $W$. For more details we refer to [17, Kap. 14].

We pick $\mu \in \Lambda^{+}$such that $\lambda=w \cdot \mu=w(\mu+\rho)-\rho$ for some $w \in W$, write

$$
S:=B_{\mu}^{0}:=\left\{\alpha \in \Delta \mid\left\langle\mu+\rho, \alpha^{\vee}\right\rangle=0\right\}
$$

and let $W_{S}$ be the subgroup of $W$ generated by all $s_{\alpha}, \alpha \in S$. Hence, $W_{S}$ coincides with the stabilizer $\left\{w^{\prime} \in W: w^{\prime} \cdot \mu=\mu\right\}$ according to [17, 2.5]. Let $W^{S}$ be the unique system of representatives of maximal length for the left cosets in $W / W_{S}$. Since $W \cdot \mu=W^{S} \cdot \mu$ we may and will assume that $w \in W^{S}$.

Theorem 5.6. The module $L(\lambda)=L(w \cdot \mu)$ has the dimension

$$
\operatorname{dim}_{U(\mathfrak{g})} L(w \cdot \mu)=\# \Phi^{+}-m_{w}
$$

where $m_{w}$ denotes the degree of the polynomial $\tilde{f}_{w}^{\Lambda}$.

Proof. Since $w \in W^{S}$, we have $B_{\mu}^{0}=S \subset \tau_{\Lambda}(w)$ according to [17, 2.7(1)] (and in the notation of loc. cit.). We may therefore apply [17, Satz 9.12] to obtain $\operatorname{dim}_{U(\mathfrak{g})} L(w \cdot \mu)=\# \Phi^{+}-m_{w}$. Note that all arguments extend from the split semisimple case of loc. cit. to the more general split reductive case considered here and that Gelfand-Kirillov dimension may be replaced by canonical dimension. 
Remark. The wish to explicitly compute the polynomial $\tilde{f}_{w}^{\Lambda}$ and its degree led to the formulation of the so-called Kazhdan-Lusztig conjecture [20]. This conjecture is now a theorem thanks to the work of Beilinson-Bernstein [4] and Brylinski-Kashiwara [7.

\section{Application to equivariant line Bundles on Drinfeld'S UPPER HALF SPACE}

In this section we explain briefly how the results of the preceding sections combined with a theorem from 28] allow to compute the dimension of representations coming from line bundles on Drinfeld's half space.

We let $\mathbf{G}=\mathrm{GL}_{d+1}$. Moreover, $\mathbf{B} \subset \mathrm{GL}_{d+1}$ equals the Borel subgroup of lower triangular matrices and $\mathbf{T} \subset \mathbf{B}$ the diagonal torus. For a decomposition $\left(n_{1}, \ldots, n_{s}\right)$ of $d+1$ the symbol $\mathbf{P}_{n_{1}, \ldots, n_{s}}$ denotes the corresponding lower standard parabolic subgroup of $\mathrm{GL}_{d+1}$ with Levi subgroup $\mathbf{L}_{n_{1}, \ldots, n_{s}}$.

Let $\mathcal{X}$ be Drinfeld's half space of dimension $d \geqslant 1$ over $K$. This is a rigid-analytic variety over $K$ given by the complement of all $K$-rational hyperplanes in projective space $\mathbb{P}_{K}^{d}$, i.e.,

$$
\mathcal{X}=\mathbb{P}_{K}^{d} \backslash \bigcup_{H \varsubsetneqq K^{d+1}} \mathbb{P}(H),
$$

where $H$ runs over the set of $K$-rational hyperplanes in $K^{d+1}$. There is a natural action of $G=\mathrm{GL}_{d+1}(K)$ on $\mathcal{X}$ induced by the algebraic action $m: \mathbf{G} \times \mathbb{P}_{K}^{d} \rightarrow \mathbb{P}_{K}^{d}$ of $\mathbf{G}$ defined by

$$
g \cdot\left[q_{0}: \cdots: q_{d}\right]:=m\left(g,\left[q_{0}: \cdots: q_{d}\right]\right):=\left[q_{0}: \cdots: q_{d}\right] g^{-1} .
$$

Let $s \in \mathbb{Z}$ and denote by $\lambda^{\prime}=(s, \ldots, s) \in \mathbb{Z}^{d}$ the constant integral weight for $\mathrm{GL}_{d}$. Let $r=\lambda_{0} \in \mathbb{Z}$ and set

$$
\lambda=(r, s, \ldots, s) \in \mathbb{Z}^{d+1} .
$$

We denote by $\mathcal{L}_{\lambda}$ the homogeneous line bundle on $\mathbb{P}_{K}^{d}=\mathrm{GL}_{d+1} / \mathbf{P}_{1, d}$ such that its fiber in the base point is the irreducible algebraic $\mathbf{L}_{1, d}$-representation corresponding to $\lambda$. Then we obtain $\mathcal{L}_{\lambda}=\mathcal{O}(r-s)$ where the $\mathbf{G}$-linearization is given by the tensor product of the natural one on $\mathcal{O}(r-s)$ with $\operatorname{det}^{s}$. The space of global sections $H^{0}\left(\mathcal{X}, \mathcal{L}_{\lambda}\right)$ is a coadmissible $D(G)$-module. We may compute its dimension as follows.

Put $w_{j}:=s_{j} \cdots s_{1}$, where $s_{i} \in W$ is the (standard) simple reflection in the Weyl group $W \cong S_{d+1}$ of $G$. Recall that . denotes the dot action of $W$ on $X^{*}(\mathbf{T})_{\mathbb{Q}}$. There is at most one integer $0 \leqslant i_{0} \leqslant d$, such that $H^{i_{0}}\left(\mathbb{P}_{K}^{d}, \mathcal{L}_{\lambda}\right)$ is nonvanishing which is $i_{0}=0$ for $r \geqslant s$ resp. $i_{0}=d$ for $s \geqslant r+d+1$. Otherwise, there is a unique integer $i_{0}<d$ with $w_{i_{0}} \cdot \lambda=w_{i_{0}+1} \cdot \lambda$. This is the case for $0 \leqslant i_{0}=s-r-1<d+1$. We write

$$
\mu_{i, \lambda}:=\left\{\begin{array}{cc}
w_{i-1} \cdot \lambda & : i \leqslant i_{0} \\
w_{i} \cdot \lambda & : i>i_{0}
\end{array}\right\}, i=1, \ldots, d .
$$

This is a $\mathbf{L}_{(\mathbf{i}, \mathbf{d}-\mathbf{i}+\mathbf{1})}$-dominant weight with respect to the Borel subgroup $\mathbf{L}_{(\mathbf{i}, \mathbf{d}-\mathbf{i}+\mathbf{1})} \cap \mathbf{B}^{+}$where $\mathbf{B}^{+}$denotes the upper triangular matrices in $\mathrm{GL}_{d+1}$. Consider the block matrix

$$
z_{j}:=\left(\begin{array}{cc}
0 & I_{j} \\
I_{d+1-j} & 0
\end{array}\right) \in G
$$

where $I_{k} \in \mathrm{GL}_{k}(K)$ denotes the $k \times k$-identity matrix. We may regard $z_{j}$ as an element of $W$ and consider the weights $z_{j}^{-1} \cdot \mu_{j, \lambda}$ for any $j=0, \ldots, d-1$. For each 
$j$ we choose an element $v_{j} \in W$ such that

$$
v_{j}^{-1} \cdot\left(z_{j}^{-1} \cdot \mu_{j, \lambda}\right) \in \Lambda^{+} \text {. }
$$

As explained in the discussion following (5.5), we can and will here assume additionally that $v_{j}$ lies in the subset $W^{S_{j}} \subset W$ corresponding to

$$
S_{j}:=B_{v_{j}^{-1} \cdot\left(z_{j}^{-1} \cdot \mu_{j, \lambda}\right)}^{0} .
$$

Theorem 6.1. The coadmissible $D(G)$-module $H^{0}\left(\mathcal{X}, \mathcal{L}_{\lambda}\right)$ has the dimension

$$
\operatorname{dim} H^{0}\left(\mathcal{X}, \mathcal{L}_{\lambda}\right)=\# \Phi^{+}-\min _{j=0, \ldots, d-1} m_{v_{j}} .
$$

Here, $m_{v_{j}}$ denotes the degree of the polynomial $\tilde{f}_{v_{j}}^{\Lambda}$.

Proof. We abbreviate $\mathcal{L}(\mathcal{X}):=H^{0}\left(\mathcal{X}, \mathcal{L}_{\lambda}\right)$. For $j=0, \ldots, d-1$ the $U(\mathfrak{g})$ module $L\left(z_{j}^{-1} \cdot \mu_{j, \lambda}\right)$ lies in the category $\mathcal{O}_{\mathrm{alg}}^{\mathfrak{p}_{j+1, d-j}}$. By [28, 5.3] the $D(G)$-module $\mathcal{F}_{P_{(j+1, d-j)}}^{G}\left(L\left(z_{j}^{-1} \cdot \mu_{j, \lambda}\right)\right)$ is simple, but we do not need this. In any case, there is a filtration of $\mathcal{L}(\mathcal{X})$ by coadmissible $D(G)$-submodules

$$
\mathcal{L}(\mathcal{X})=\mathcal{L}(\mathcal{X})^{0} \supset \mathcal{L}(\mathcal{X})^{1} \supset \cdots \supset \mathcal{L}(\mathcal{X})^{d-1} \supset \mathcal{L}(\mathcal{X})^{d}=H^{0}\left(\mathbb{P}^{d}, \mathcal{L}\right),
$$

such that for $j=0, \ldots, d-1$, there are $D(G)$-module extensions

$$
0 \rightarrow \mathcal{F}_{P_{(j+1, d-j)}^{G}}^{G}\left(L\left(z_{j}^{-1} \cdot \mu_{j, \lambda}\right)\right) \rightarrow\left(\mathcal{L}(\mathcal{X})^{j} / \mathcal{L}(X)^{j+1}\right) \rightarrow \mathcal{Z}_{j} \rightarrow 0
$$

where $\mathcal{Z}_{j}$ is dual to a locally algebraic $G$-representation; cf. [28, Cor. 7.6] and [27. Cor. 2.2.9]. Since $\mathcal{Z}_{j}$ has dimension zero, we obtain

$$
\operatorname{dim}(\mathcal{L}(\mathcal{X}))=\max _{j=0, \ldots, d-1} \operatorname{dim} \mathcal{F}_{P_{(j+1, d-j)}^{G}}^{G}\left(L\left(z_{j}^{-1} \cdot \mu_{j, \lambda}\right)\right)
$$

and so Theorem 5.6 yields the assertion.

\section{Dimensions for $\mathrm{GL}_{2}\left(\mathbb{Q}_{p}\right)$ And $p$-Adic Galois Representations}

Let $L=\mathbb{Q}_{p}$ in this section. We first discuss some preliminaries concerning locally analytic vectors in Banach space representations. In the second part we apply this to representations of $\mathrm{GL}_{2}\left(\mathbb{Q}_{p}\right)$ occuring in Colmez' p-adic Langlands correspondence [12].

We consider an arbitrary locally analytic group $G$ which has dimension $d$. Fix some arbitrary compact open subgroup $H \subset G$ and let

$$
o_{K}[[H]]:=\lim _{N \triangleleft H} o_{K}[H / N]
$$

be the completed group ring of $H$ with coefficients in $o_{K}$. Write

$$
K[[H]]:=K \otimes_{o_{K}} o_{K}[[H]] \text { and } k[[H]]:=k \otimes_{o_{K}} o_{K}[[H]]
$$

where $k:=o_{K} /(\pi)$ is the residue field of $o_{K}$. These rings are noetherian and, in case $H$ has no element of order $p$, even Auslander regular with global dimensions $\operatorname{gld}\left(o_{K}[[H]]\right)=d+1$ and $\operatorname{gl} d(k[[H]])=\operatorname{gld}(K[[H]])=d$ [43, Thm. 3.29], [2, Thm. $5.1 / 5.2]$. We assume in the following that $H$ has no element of order $p$. If $R$ denotes one of the rings $o_{K}, K$ or $k$, we define the category of coadmissible modules over $R$ to be the category of finitely generated $R[[H]]$-modules $M$ equipped with a compatible linear action of $G$ (and obvious morphisms). It is abelian and independent of the 
choice of $H$. If $H^{\prime} \subset H$ is a subgroup one has $j_{R\left[\left[H^{\prime}\right]\right]}(M)=j_{R[[H]]}(M)$ along the lines of [41, Lem. 8.8]. The number

$$
\operatorname{dim}_{R[[H]]} M=\operatorname{gld}(R[[H]])-j_{R[[H]]}(M)
$$

is therefore independent of the particular choice of $H$ in $G$. If $M$ is additionally flat over $o_{K}$, we then have

$$
\operatorname{dim}_{K[[H]]} K \otimes_{o_{K}} M=\operatorname{dim}_{k[[H]]} k \otimes_{o_{K}} M=\operatorname{dim}_{o_{K}[[H]]} M-1
$$

according to [3, Thm. 3.3 and Lem. 8.5]. In case of $R=K$ we simply write dim instead of $\operatorname{dim}_{K[[H]]}$ in the following.

Passage to the continuous dual $V \rightarrow V^{\prime}$ induces an anti-equivalence of the category of admissible Banach space representations of $G$ with the coadmissible modules over $K$ [39, Thm. 3.5]. The restriction of this equivalence to unitary representations is compatible via reduction mod $\pi$ with Pontryagin duality $(\cdot)^{\vee}$ between admissible smooth representations over $k$ and the coadmissible modules over $k$ 29, Lem. 5.4]. Given a unitary representation, one may choose an open bounded $G$-invariant lattice $V^{0}$ in $V$ and consider its reduction $\bar{V}:=V^{0} / \pi V^{0}$. Finally, passage to locally analytic vectors $V \mapsto V^{a n}$ is an exact functor from admissible Banach space representations to admissible locally analytic representations [41, §7].

Proposition 7.2. One has $\operatorname{dim} V^{\prime}=\operatorname{dim}\left(V^{a n}\right)^{\prime}$ for any admissible Banach space representation $V$ of $G$. If $V$ is unitary with a chosen reduction $\bar{V}$, then $\operatorname{dim} V^{\prime}=$ $\operatorname{dim}_{k[[H]]} \bar{V}^{\vee}$.

Proof. As representations of $H$, the functor $V \mapsto V^{a n}$ is given on the level of coadmissible modules as the base change $M \mapsto D(H) \otimes_{K[[H]]} M$. Since the homomorphism $K[[H]] \rightarrow D(H)$ is faithfully flat 41, Thm. 5.2], the first assertion follows from Lemma 2.2. The second assertion follows from our discussion above.

For $V$ an admissible Banach space representation or locally analytic representation we will write in the following $\operatorname{dim} V$ for the dimension of the dual $V^{\prime}$.

We now specialize to certain reductive groups $G$. To this end, let $\mathbf{G}$ be a connected split reductive group scheme over $o_{L}$ with Lie algebra $\mathfrak{g}$. Let $\bar{\kappa}$ be an algebraic closure of $\kappa$, the residue field of $o_{L}$. Let us consider the following three hypothesis on the geometric closed fiber $\mathbf{G}_{\bar{s}}=\mathbf{G} \otimes_{o_{L}} \bar{\kappa}$ of $\mathbf{G}$ which are familiar from the theory of modular Lie algebras (cf. [18, 6.3).

(H1) The derived group of $\mathbf{G}_{\bar{s}}$ is (semisimple) simply connected.

(H2) The prime $p$ is good for the $\bar{\kappa}$-Lie algebra $\operatorname{Lie}\left(\mathbf{G}_{\bar{s}}\right)$.

(H3) There exists a $\mathbf{G}_{\bar{s}}$-invariant nondegenerate bilinear form on $\operatorname{Lie}\left(\mathbf{G}_{\bar{s}}\right)$.

For example, the general linear group $\mathrm{GL}_{n}$ satisfies these conditions for all primes $p$ (using the trace form for $(\mathrm{H} 3)$ ). Any almost simple and simply connected $\mathbf{G}_{\bar{s}}$ satisfies these conditions if $p \geqslant 7$ (and if $p$ does not divide $n+1$ if $\mathbf{G}_{\bar{s}}$ is of type $\left.A_{n}\right)$. For a more detailed discussion of these conditions we refer to loc. cit.

We assume from now on that (H1)-(H3) hold. As before, $\Phi^{+}$denotes a set of positive roots of $\mathbf{G}$ and the number $r$ denotes half the dimension of the minimal nilpotent coadjoint orbit of $\mathbf{G}_{\bar{s}}$ (8], Rem. 4.3.4). We assume from now on that the Lie algebra of our locally analytic group $G$ is isomorphic to $\mathfrak{g}_{L}:=L \otimes_{o_{L}} \mathfrak{g}$.

Proposition 7.3. Let $V$ be an admissible $G$-Banach space representation. If $V^{a n}$ has an infinitesimal character, then $\operatorname{dim} V \leqslant 2 \cdot \# \Phi^{+}$. If $\operatorname{dim} V \geqslant 1$, then $\operatorname{dim} V \geqslant r$. 
Proof. The second statement follows from the main result of [3], extended to reductive groups satisfying (H1)-(H3) in [36. Suppose now that $V^{a n}$ has an infinitesimal character. By [36, 9.4/9.6] it suffices to show that, for any $n \geqslant 1$, the dimension of a finitely generated module $M$ over the Auslander regular ring $\widehat{U(\mathfrak{g})}{ }_{n, K}$ with infinitesimal character is bounded above by $2 \cdot \# \Phi^{+}$. Here, $\left.\widehat{U(\mathfrak{g})}\right)_{n, K}$ denotes the $\pi$-adic completion (with subsequent inversion of $\pi$ ) of the universal enveloping algebra $U\left(\pi_{L}^{n} \mathfrak{g}\right)$ for a choice of uniformizer $\pi_{L}$ of $o_{L}$. We may choose a good double filtration for $M$ and form its double graded module $\operatorname{Gr}(M)$ in the sense of [3, 3.2]. The latter is a finitely generated module over $\operatorname{Gr}\left(\widehat{U(\mathfrak{g})_{n, K}}\right)=\operatorname{Sym}\left(\mathfrak{g}_{\kappa}\right)$ whose support has dimension equal to the dimension of $M$. Since $M$ has a central character, $\operatorname{Gr}(M)$ is annihilated by $\operatorname{Sym}\left(\mathfrak{g}_{\kappa}\right)_{+}^{\mathbf{G}_{k}}$, the ideal of invariant polynomials without constant term. Its support lies therefore in the cone of nilpotent elements of $\mathfrak{g}_{\kappa}$ which has dimension $2 \cdot \# \Phi^{+}$.

We recall that the representation $V^{a n}$ always admits an infinitesimal character, if $G=\mathrm{GL}_{2}\left(\mathbb{Q}_{p}\right)$ and if the representation $V$ is absolutely irreducible [13, introduction, Thm. 3]. Because [13] assumes $p>3$ (which is in turn caused by relying on [30]), we will do so here also and throughout the rest of this section.

We now let $\mathbf{G}=\mathrm{GL}_{2}$ and $L=\mathbb{Q}_{p}$. Moreover, $B \subset G$ denotes the Borel subgroup of upper triangular matrices. We fix a finite extension $\mathbb{Q}_{p} \subseteq K$ as a coefficient field for the representations. We denote the continuous character $x \mapsto x|x|$ of $\mathbb{Q}_{p}^{\times}$by $\chi$. Finally, $\operatorname{Gal}\left(\overline{\mathbb{Q}}_{p} / \mathbb{Q}_{p}\right)$ denotes the absolute Galois group of $\mathbb{Q}_{p}$. In 12 Colmez establishes a correspondence

$$
V \mapsto \Pi(V)
$$

from absolutely irreducible 2-dimensional representations $V$ of $\operatorname{Gal}\left(\overline{\mathbb{Q}}_{p} / \mathbb{Q}_{p}\right)$ over $K$ to absolutely irreducible unitary admissible $G$-representations. This correspondence is based on the construction of a $G$-representation $D(V) \otimes \mathbb{P}^{1}$ attached to $V$ with central character $\delta(x)=\chi^{-1} \operatorname{det}_{V}(x)$ where $\operatorname{det}_{V}$ is the character of $\mathbb{Q}_{p}^{\times}$ corresponding by local class field to the determinant of $V$. The representation $D(V) \otimes \mathbb{P}^{1}$ is an extension of $\Pi(V)$ by its dual twisted by $\delta \circ$ det. In particular, the central character of $\Pi(V)$ equals $\delta$ and Proposition 7.3 implies $\operatorname{dim} \Pi(V) \leqslant 2$. Let us determine $\operatorname{dim} \Pi(V)$ in case $\Pi(V)$ belongs to the unitary principal series, i.e., in case $V$ is trianguline 11 .

In the following, all $(\varphi, \Gamma)$-modules are taken over the classical Robba ring $\mathscr{R}$. Given a continuous character $\eta: \mathbb{Q}_{p}^{\times} \rightarrow K^{\times}$, the associated $(\varphi, \Gamma)$-module of rank 1 is denoted by $\mathscr{R}(\eta)$. Recall that a 2 -dimensional Galois representation is called trianguline, if the associated étale $(\varphi, \Gamma)$-module is an extension of two (non-étale if $V$ is irreducible) modules of rank 1. If $\eta_{1}, \eta_{2}$ are two continuous characters $\mathbb{Q}_{p}^{\times} \rightarrow K^{\times}$, we denote the locally analytic induction $\operatorname{Ind}_{B}^{G}\left(\eta_{2} \otimes \eta_{1} \chi^{-1}\right)$ simply by $B^{a n}\left(\eta_{1}, \eta_{2}\right)$ (note the reversed order of the $\eta_{i}$ ).

Proposition 7.4. One has $\operatorname{dim} \Pi(V)=1$ for any absolutely irreducible 2-dimensional representation $V$ which is trianguline.

Proof. Let $\Delta(s)$ be the étale $(\varphi, \Gamma)$-module associated with $V$. Here, $s=\left(\delta_{1}, \delta_{2}, \mathscr{L}\right)$ is the associated parameter consisting of continuous characters $\delta_{1}, \delta_{2}: \mathbb{Q}_{p}^{\times} \rightarrow K^{\times}$ and an element $\mathscr{L} \in \mathbb{P}\left(\operatorname{Ext}^{1}\left(\mathscr{R}\left(\delta_{1}\right), \mathscr{R}\left(\delta_{2}\right)\right)\right)$. In [10, Thm. 0.7] (compare also 25]) the locally analytic representation $\Pi(V)^{a n}$ is computed. Either we have the exact 
sequence of locally analytic $G$-representations

$$
0 \rightarrow B^{a n}\left(\delta_{1}, \delta_{2}\right) \rightarrow \Pi(V)^{a n} \rightarrow B^{a n}\left(\delta_{2}, \delta_{1}\right) \rightarrow 0
$$

(the generic case) or we have an exact sequence of locally analytic $G$-representations

$$
0 \rightarrow E_{\mathscr{L}} \rightarrow \Pi(V)^{a n} \rightarrow B^{a n}\left(\delta_{2}, \delta_{1}\right) \rightarrow 0
$$

(the special case) where $E_{\mathscr{L}}$ is an extension of a representation $W\left(\delta_{1}, \delta_{2}\right)$ on a finite-dimensional $K$-vector space by $\mathrm{St}^{a n}\left(\delta_{1}, \delta_{2}\right)$. Here, $W\left(\delta_{1}, \delta_{2}\right)$ is in fact a subrepresentation of $B^{a n}\left(\delta_{1}, \delta_{2}\right)$ and $\mathrm{St}^{a n}\left(\delta_{1}, \delta_{2}\right)$ denotes the corresponding quotient of $B^{a n}\left(\delta_{1}, \delta_{2}\right)$. We have $\operatorname{dim} B^{a n}\left(\eta_{1}, \eta_{2}\right)=1$ according to Corollary 4.12 for any pair of continuous characters $\left(\eta_{1}, \eta_{2}\right)$ which settles the generic case. Since $\operatorname{dim} W\left(\delta_{1}, \delta_{2}\right)=0$ we have $\operatorname{dim} E_{\mathscr{L}}=1$ and this settles the special case.

Corollary 7.5. One has $\operatorname{dim} \Pi(V)=1$ for any absolutely irreducible 2-dimensional representation $V$.

Proof. Any smooth principal series representation $P S(\bar{\chi})$ of $G$ over $k$ may be lifted to a continuous principal series representation $P S(\chi)$ over $K$ by taking a unitary lift $\chi$ of the inducing smooth character $\bar{\chi}$. Since $P S(\chi)^{a n}$ equals the locally analytic induction of $\chi\left[38\right.$, we obtain $\operatorname{dim}_{k[[H]]} P S(\bar{\chi})^{\vee}=1$ according to Corollary 4.12 and Propositon 7.2. On the other hand, consider an absolutely irreducible supersingular representation $W$ of $G$ over $k$. Up to a twist with a smooth character, $W$ is isomorphic to a representation $\pi(r, 0,1)$ with $r \in\{0, \ldots, p-1\}$, in the notation of [6. Def. 1.1]. According to [6, Thm. 1.4(i)] we have $\pi(r, 0,1)=\overline{\Pi(V)}$ with a crystalline (hence trianguline) representation $V$. Since twisting with characters preserves dimension, we obtain $\operatorname{dim}_{k[[H]]} W=1$ according to Propositions 7.2 and 7.4 Now let $V$ be any absolutely irreducible 2-dimensional representation of $\operatorname{Gal}\left(\overline{\mathbb{Q}}_{p} / \mathbb{Q}_{p}\right)$. According to [9, Thm. 1.4] (proved for $p \geqslant 5$ already in [30]) the reduction $\overline{\Pi(V)}$ is, up to a finite extension of $K$, either absolutely irreducible supersingular or of finite length with composition factors which occur as composition factors in certain principal series representations. By Lemma 2.2 passing to finite extensions does not change dimensions. Hence $\operatorname{dim} \overline{\Pi(V)}^{\vee}=1$ by our initial remarks and then $\operatorname{dim} \Pi(V)=1$ by Proposition 7.2 ,

\section{ACKNOWLEDGMEnTs}

We would like to thank Vytautas Paškūnas for his remarks and for communicating to us the idea of the proof of Corollary 7.5. We would also like to thank the anonymous referee for helpful comments and corrections.

\section{REFERENCES}

[1] K. Ardakov, $\hat{\mathcal{D}}$-modules on rigid analytic spaces, Proc. International Congress of Mathematicians 2014 (to appear).

[2] K. Ardakov and K. A. Brown, Ring-theoretic properties of Iwasawa algebras: a survey, Doc. Math. Extra Vol. (2006), 7-33. MR2290583 (2007k:11185)

[3] K. Ardakov and S. Wadsley, On irreducible representations of compact p-adic analytic groups, Ann. of Math. (2) 178 (2013), no. 2, 453-557, DOI 10.4007/annals.2013.178.2.3. MR3071505

[4] A. Beilinson and J. Bernstein, Localisation de g-modules (French, with English summary), C. R. Acad. Sci. Paris Sér. I Math. 292 (1981), no. 1, 15-18. MR610137 (82k:14015)

[5] P. Berthelot, D-modules arithmétiques. I. Opérateurs différentiels de niveau fini (French, with English summary), Ann. Sci. École Norm. Sup. (4) 29 (1996), no. 2, 185-272. MR 1373933 (97b:14019) 
[6] C. Breuil, Sur quelques représentations modulaires et p-adiques de $\mathrm{GL}_{2}\left(\mathbf{Q}_{p}\right)$. II (French, with French summary), J. Inst. Math. Jussieu 2 (2003), no. 1, 23-58, DOI 10.1017/S1474748003000021. MR1955206 (2005d:11079)

[7] J.-L. Brylinski and M. Kashiwara, Kazhdan-Lusztig conjecture and holonomic systems, Invent. Math. 64 (1981), no. 3, 387-410, DOI 10.1007/BF01389272. MR632980 (83e:22020)

[8] D. H. Collingwood and W. M. McGovern, Nilpotent orbits in semisimple Lie algebras, Van Nostrand Reinhold Mathematics Series, Van Nostrand Reinhold Co., New York, 1993. MR.1251060 (94j:17001)

[9] P. Colmez, G. Dospinescu, and V. Paškūnas, The p-adic local Langlands correspondence for $\mathrm{GL}_{2}\left(\mathbb{Q}_{p}\right)$, Camb. J. Math. 2 (2014), no. 1, 1-47, DOI 10.4310/CJM.2014.v2.n1.a1. MR 3272011

[10] P. Colmez, La série principale unitaire de $\mathrm{GL}_{2}\left(\mathbf{Q}_{p}\right)$ : vecteurs localement analytiques, Automorphic Forms and Galois Representations, Vol. 1, London Math. Soc. Lect. Note Series 415 (2014), 286-358.

[11] P. Colmez, La série principale unitaire de $\mathrm{GL}_{2}\left(\mathbf{Q}_{p}\right)$ (French, with English and French summaries), Astérisque 330 (2010), 213-262. MR2642407 (2011g:22026)

[12] P. Colmez, Représentations de $\mathrm{GL}_{2}\left(\mathbf{Q}_{p}\right)$ et $(\phi, \Gamma)$-modules, Astérisque (2010), no. 330, 281509.

[13] G. Dospinescu, Actions infinitésimales dans la correspondance de Langlands locale $p$ adique (French, with English summary), Math. Ann. 354 (2012), no. 2, 627-657, DOI 10.1007/s00208-011-0736-2. MR2965255

[14] M. Emerton, Locally analytic vectors in representations of locally p-adic analytic groups, Preprint. To appear in: Memoirs of the AMS.

[15] A. Ya. Helemskii, Banach and locally convex algebras, Oxford Science Publications, The Clarendon Press, Oxford University Press, New York, 1993. Translated from the Russian by A. West. MR1231796 (94f:46001)

[16] J. E. Humphreys, Representations of semisimple Lie algebras in the BGG category $\mathcal{O}$, Graduate Studies in Mathematics, vol. 94, American Mathematical Society, Providence, RI, 2008. MR2428237 (2009f:17013)

[17] J. C. Jantzen, Einhüllende Algebren halbeinfacher Lie-Algebren (German), Ergebnisse der Mathematik und ihrer Grenzgebiete (3) [Results in Mathematics and Related Areas (3)], vol. 3, Springer-Verlag, Berlin, 1983. MR.721170 (86c:17011)

[18] J. C. Jantzen, Representations of Lie algebras in prime characteristic, Representation theories and algebraic geometry (Montreal, PQ, 1997), NATO Adv. Sci. Inst. Ser. C Math. Phys. Sci., vol. 514, Kluwer Acad. Publ., Dordrecht, 1998, pp. 185-235. Notes by Iain Gordon. MR.1649627(99h:17026)

[19] A. Joseph, Goldie rank in the enveloping algebra of a semisimple Lie algebra. I, II, J. Algebra 65 (1980), no. 2, 269-283, 284-306, DOI 10.1016/0021-8693(80)90217-3. MR585721 (82f:17009)

[20] D. Kazhdan and G. Lusztig, Representations of Coxeter groups and Hecke algebras, Invent. Math. 53 (1979), no. 2, 165-184, DOI 10.1007/BF01390031. MR560412 (81j:20066)

[21] J. Lepowsky, Generalized Verma modules, the Cartan-Helgason theorem, and the HarishChandra homomorphism, J. Algebra 49 (1977), no. 2, 470-495. MR0463360 (57 \#3312)

[22] T. Levasseur, Some properties of noncommutative regular graded rings, Glasgow Math. J. 34 (1992), no. 3, 277-300, DOI 10.1017/S0017089500008843. MR.1181768 (93k:16045)

[23] H. Li and F. van Oystaeyen, Zariskian filtrations, K-Monographs in Mathematics, vol. 2, Kluwer Academic Publishers, Dordrecht, 1996. MR,1420862 (97m:16083)

[24] H. Li, Lifting Ore sets of Noetherian filtered rings and applications, J. Algebra 179 (1996), no. 3, 686-703, DOI 10.1006/jabr.1996.0031. MR1371738 (97a:16088)

[25] R. Liu, B. Xie, and Y. Zhang, Locally analytic vectors of unitary principal series of $\mathrm{GL}_{2}\left(\mathbb{Q}_{p}\right)$ (English, with English and French summaries), Ann. Sci. Éc. Norm. Supér. (4) 45 (2012), no. 1, 167-190. MR2961790

[26] J. C. McConnell and J. C. Robson, Noncommutative Noetherian rings, Pure and Applied Mathematics (New York), John Wiley \& Sons, Ltd., Chichester, 1987. With the cooperation of L. W. Small; A Wiley-Interscience Publication. MR934572 (89j:16023)

[27] S. Orlik, Equivariant vector bundles on Drinfeld's upper half space, Invent. Math. 172 (2008), no. 3, 585-656, DOI 10.1007/s00222-008-0112-3. MR2393081 (2009c:22019) 
[28] S. Orlik and M. Strauch, On Jordan-Hölder series of some locally analytic representations, J. Amer. Math. Soc. 28 (2015), no. 1, 99-157, DOI 10.1090/S0894-0347-2014-00803-1. MR.3264764

[29] V. Paškūnas, Admissible unitary completions of locally $\mathbb{Q}_{p}$-rational representations of $\mathrm{GL}_{2}(F)$, Represent. Theory 14 (2010), 324-354, DOI 10.1090/S1088-4165-10-00373-0. MR.2608966 (2011i:22017)

[30] V. Paškūnas, The image of Colmez's Montreal functor, Publ. Math. Inst. Hautes Études Sci. 118 (2013), 1-191, DOI 10.1007/s10240-013-0049-y. MR3150248

[31] G. S. Rinehart, Differential forms on general commutative algebras, Trans. Amer. Math. Soc. 108 (1963), 195-222. MR0154906 (27 \#4850)

[32] T. Schmidt, Auslander regularity of p-adic distribution algebras, Represent. Theory 12 (2008), 37-57, DOI 10.1090/S1088-4165-08-00323-3. MR2375595 (2009b:22018)

[33] T. Schmidt, Analytic vectors in continuous p-adic representations, Compos. Math. 145 (2009), no. 1, 247-270, DOI 10.1112/S0010437X08003825. MR2480502(2009k:22025)

[34] T. Schmidt, Stable flatness of nonarchimedean hyperenveloping algebras, J. Algebra 323 (2010), no. 3, 757-765, DOI 10.1016/j.jalgebra.2009.11.018. MR2574862 (2011a:17023)

[35] T. Schmidt, Verma modules over p-adic Arens-Michael envelopes of reductive Lie algebras, J. Algebra 390 (2013), 160-180, DOI 10.1016/j.jalgebra.2013.04.038. MR.3072116

[36] T. Schmidt, On locally analytic Beilinson-Bernstein localization and the canonical dimension, Math. Z. 275 (2013), no. 3-4, 793-833, DOI 10.1007/s00209-013-1161-x. MR3127038

[37] P. Schneider, Nonarchimedean functional analysis, Springer Monographs in Mathematics, Springer-Verlag, Berlin, 2002. MR1869547 (2003a:46106)

[38] P. Schneider, Continuous representation theory of p-adic Lie groups, International Congress of Mathematicians. Vol. II, Eur. Math. Soc., Zürich, 2006, pp. 1261-1282.

[39] P. Schneider and J. Teitelbaum, Banach space representations and Iwasawa theory, Israel J. Math. 127 (2002), 359-380, DOI 10.1007/BF02784538. MR1900706 (2003c:22026)

[40] P. Schneider and J. Teitelbaum, Locally analytic distributions and p-adic representation theory, with applications to $\mathrm{GL}_{2}$, J. Amer. Math. Soc. 15 (2002), no. 2, 443-468 (electronic), DOI 10.1090/S0894-0347-01-00377-0. MR.1887640 (2003b:11132)

[41] P. Schneider and J. Teitelbaum, Algebras of $p$-adic distributions and admissible representations, Invent. Math. 153 (2003), no. 1, 145-196, DOI 10.1007/s00222-002-0284-1. MR.1990669 (2004g:22015)

[42] P. Schneider and J. Teitelbaum, Duality for admissible locally analytic representations, Represent. Theory 9 (2005), 297-326 (electronic), DOI 10.1090/S1088-4165-05-00277-3. MR2133762(2006a:22016)

[43] O. Venjakob, On the structure theory of the Iwasawa algebra of a p-adic Lie group, J. Eur. Math. Soc. (JEMS) 4 (2002), no. 3, 271-311, DOI 10.1007/s100970100038. MR.1924402 (2004h:16029)

Institute de Recherche Mathématique de Rennes, Université de Rennes 1, Campus de

Beaulieu, 35042 Rennes, France

E-mail address: Tobias.Schmidt@univ-rennes1.fr

Department of Mathematics, Indiana University, Rawles Hall, Bloomington, Indiana 47405

E-mail address: mstrauch@indiana.edu 